\title{
Local Relativistic Invariant Flows for Quantum Fields
}

\author{
S. Albeverio ${ }^{1}$, Ph. Blanchard ${ }^{2}$, Ph. Combe ${ }^{3}$, R. Høegh-Krohn ${ }^{4}$, and M. Sirugue ${ }^{5}$ \\ 1 Mathematisches Institut, Ruhr-Universität, D-4630 Bochum, Federal Republic of Germany \\ 2 Fakultät für Physik, Universität Bielefeld, D-4800 Bielefeld, Federal Republic of Germany \\ 3 Centre de Physique Théorique, CNRS, Marseille and \\ Université d'Aix-Marseille II, Marseille, France \\ 4 Matematisk Institutt, Universitetet i Oslo, Oslo, Norway and \\ Université de Provence et CNRS-Marseille, Marseille, France \\ 5 Centre de Physique Théorique, CNRS, Marseille, France
}

\begin{abstract}
For quantum fields with trigonometric interaction in arbitrary space dimension we construct a representation of the Lorentz group by automorphisms on a Banach space generated by the Weyl algebra.
\end{abstract}

\section{Introduction}

It is well known that for systems with infinitely many degrees of freedom the problem of the formulation of the dynamics is intimately connected with the "kinematical problem" of finding a suitable representation of the basic operators of the theory. The problem as stated above has been discussed both in physical and mathematical works, see e.g. the basic early work [20, 21, 23, 24, 29, 34]. From a mathematical point of view the problem has been seen as the one connected with the phenomenon of equivalence versus singularity of measures on function space, and has been discussed extensively in this way, see e.g. [26, 45, 48]. Nevertheless, despite this basic physical and mathematical problem, since the very beginnings of quantum field theory, the canonical formalism has been for a long time the most fascinating point of reference (see e.g. Dirac [18] and especially Heisenberg and Pauli [28] but also Wentzel [46]). It is certainly the most ambitious program for field quantization in as much as it is an attempt to give a direct extension of the quantum mechanics of finitely many degrees of freedom to the case of systems of infinitely many degrees of freedom. The canonical program was pursued further in the late fifties especially by Coester and Haag [12], Araki [8], Klauder [31], Streater [42], and Segal [38]. The difficulties of the canonical formalism (Haag's theorem $[27,43]$, which prevents the free and interacting fields to be in the same representation of the canonical commutation relations) are well known and have to be bypassed, when starting in Fock space, by suitable limit procedures. Only in the sixties and in the last decade models satisfying the basic postulates of locality and relativistic invariance have been constructed (see any of an excellent series of surveys on constructive quantum field e.g. [41,25]), and indeed the basic 
postulates of the canonical formalism have been verified in some of these two space-time dimensional models starting with the one with trigonometric interaction [3]. On the other hand nothing of this kind is at the present time available in higher dimensions, although Wightman models have been constructed for space-time dimension three (see e.g. $[19,32]$ and references therein). For other approachs see e.g. $[9,35,38,40]$. The construction of canonical operators in the Weyl form is the problem of finding unitary operators which commute up to a factor of modulus one, hence suggests connection with the representation theory of abelian groups $[47,8,10]$.

In the present paper, our approach to the construction of quantum fields is based on the following observation. Let $H_{I}^{\kappa}$ defined by:

$$
H_{I}^{\kappa}=\int d \mu_{\kappa}(g) W^{\zeta}(g)
$$

be an interaction Hamiltonian, where $W^{\zeta}(g)$ is a measurable projective unitary representation of a group $G$, on a Hilbert space $\mathscr{H}$. Here $d \mu_{\kappa}(g)$ is a bounded measure on $G$ which depends on a positive parameter $\kappa$ (the "cut-off"). Let $H_{0}$ be the infinitesimal generator of a strongly continuous group of unitaries, which implements on $\mathscr{H}$ the one parameter group of automorphisms $t \rightarrow \alpha_{t}^{0}$ of $G$

$$
t \rightarrow W^{\zeta}\left(\alpha_{t}^{0}(g)\right)=\exp \left\{i t H_{0}\right\} W^{\zeta}(g) \exp \left\{-i t H_{0}\right\} .
$$

We assume, and this is satisfied in the applications, that in the presence of the cutoff the interaction picture is valid in the sense that one has for all $\Phi$ and $\Psi$ in $H$ :

$$
\begin{aligned}
(\Phi, & \left.\exp \left\{i H^{\kappa} t\right\} W^{\zeta}(g) \exp \left\{-i H^{\kappa} t\right\} \Psi\right) \\
= & \left(\Phi, \exp \left\{i t H^{\kappa}\right\} \exp \left\{i t H_{0}\right\} W^{\zeta}\left(\alpha_{1}^{0}(g)\right)\right. \\
& \left.\cdot \exp \left\{i t H_{0}\right\} \exp \left\{-i t H^{\kappa}\right\} \Psi\right),
\end{aligned}
$$

where $H^{\kappa}=H_{0}+H_{I}^{\kappa}$ is the total Hamiltonian. The special form (1.1) of the interaction $H_{I}^{\kappa}$ allows to rewrite the matrix element (1.3) as the expectation value of a functional of the time zero matrix elements with respect to a Poisson measure through a Feynman path formula. Furthermore this expression extends quite naturally to a suitable linear space of bounded functionals on $G$, which are no longer necessarily of the form (1.3), i.e. they are not necessarily matrix elements of a unitary projective representation of $G$ on a Hilbert space $\mathscr{H}$, and defines a one parameter group of automorphisms (flow) $\alpha_{t}^{\kappa}$ associated with the interaction $H^{\kappa}$.

A central result of this paper is that the limit for $\kappa \rightarrow \infty$ of the flow $\alpha_{t}^{\kappa}$ exists, in the case where $H_{I}^{\kappa}$ stands for an ultraviolet cut-off relativistic local interaction, on a dense subset of bounded functionals. More precisely we construct a simultaneous representation on a Banach space of the Weyl canonical commutation relations and of the Poincaré group in all space dimensions for models in which the interaction (like in the sine-Gordon model) is of the type

$$
H_{I}=\lambda \int_{\mathbb{R}^{s}} d x \int_{\mathbb{R}} d v(\alpha) \cos (\alpha \phi(x)+\theta), \quad 0 \leqq \theta \leqq 2 \pi,
$$

where $s$ is the number of space dimensions, $d v$ is an arbitrary bounded measure on $\mathbb{R}$ and $\lambda$ a real constant. Hence we get time zero fields and canonical conjugate momenta as well as a natural dynamics acting on them in the sense of 
automorphisms. This theory is a nontrivial partial solution of both the kinematical and dynamical problems alluded above. Introducing the usual renormalization procedure, it is possible to show that for $s=1, m=0$ and $\lambda$ sufficiently small, our construction coincides with the one obtained before by other means (see e.g. $[16,22])$, when interpreted in the underlying Hilbert space. We also give a solution of the Schrödinger equation, first discussed by Symanzik (see e.g. [44]) for trigonometric models. Interactions of the above form with ultra-violet cut-off have been discussed before in $[1,2,5,15,30]$. A Schrödinger equation for them was shortly pointed out in [6], but in a different form from the present one, using the definitions of Feynman path integral by normalized oscillatory integrals given in $[5]$.

This paper is organized as follows: in Sect. 2, we study both left and right regular projective representations of a group $G$ by isometric operators on the Banach space $B(G)$ of bounded functionals on $G$ (Proposition 2.1). This space contains the subspace of matrix elements (1.3) of unitary projective representations of the group $G$ as well as the dense subset generated by the characters of $G$. It is a natural space for looking at solutions of Schrödinger-like equations for the time translated matrix elements of exponentials of the field operators, which for trigonometric interactions can be expressed as Poisson expectation values (Theorem 2.5). We then define a flow on the Banach space $B(G)$ which represents the perturbed evolution in the Schrödinger picture.

In Sect. 3 we give the definition of the trigonometric interaction [30] and we derive the Schrödinger equation satisfied by the time translated functionals. We then remove the ultraviolet cut-off and the space cut-off from the solution of these equations for any space time dimension. Let us observe finally that if there existed relativistic quantum field models in higher dimensions satisfying all the Wightman axioms, and in addition some suitable technical assumptions, they would appear in our representation. For smooth initial conditions with compact support, i.e. in $\mathscr{D}\left(\mathbb{R}^{s}\right)$, we then get a relativistic flow on a dense set of functionals in the infinite volume limit, associated with time translations. We also discuss the case of initial conditions in $\mathscr{S}\left(\mathbb{R}^{s}\right)$, using methods of classical statistical mechanics (see $[1,2]$ ), obtaining the existence of the infinite volume limit for small times. We also discuss the asymptotic behaviour in time.

\section{Time Translation Automorphisms Associated with Projective Representations of a Group}

Let $G$ be a (topological) group and $\zeta$ a multiplier on $G$, i.e. a (continuous) function from $G \times G$ to the one dimensional torus such that

$$
\begin{gathered}
\zeta\left(g_{1}, g_{2}\right) \zeta\left(g_{1} \cdot g_{2}, g_{3}\right)=\zeta\left(g_{1}, g_{2} \cdot g_{3}\right) \zeta\left(g_{2}, g_{3}\right), \\
\zeta(e, e)=\zeta\left(g, g^{-1}\right)=1, \quad \forall g \in G .
\end{gathered}
$$

Let $B(G)$ be the Banach space of complex-valued bounded Borel functions on $G$ equipped with the sup-norm. We define $R^{\zeta}$ and $L^{\bar{\zeta}}$ to be the isometric projective representations of $G$ on $B(G)$ given by 


$$
\begin{aligned}
& \left(R^{\zeta}(g) F\right)(h) \equiv \zeta(h, g) F(h g), \\
& \left(L^{\bar{\zeta}}(g) F\right)(h) \equiv \zeta\left(g^{-1}, h\right) F\left(g^{-1} h\right), g, h \in G .
\end{aligned}
$$

These representations commute and moreover they allow us to implement automorphisms of $G$

where

$$
U_{\sigma}^{0} R^{\zeta}(g)\left(U_{\sigma}^{0}\right)^{-1}=R^{\zeta}(\sigma(g))
$$

$$
\left(U_{\sigma}^{0} F\right)(h) \equiv F\left(\sigma^{-1}(h)\right),
$$

for any automorphism $\sigma$ of $G$ which leaves $\zeta$ invariant. Similar formulas hold for $L^{\bar{\zeta}}$.

Let $B_{1}(G)$ be the subspace of $B(G)$ generated by the finite linear combinations of the functions

$$
h \rightarrow F_{\Phi, \Psi}^{W^{\zeta}}(h)=\left(\Phi, W^{\zeta}(h) \Psi\right),
$$

where $W^{\zeta}$ is a projective unitary representation of $G$ in a Hilbert space $\mathscr{H}$ with scalar product (, ) and $\Phi, \Psi$ are vectors in $\mathscr{H} . B_{1}(G)$ is stable with respect to both $R^{\zeta}$ and $L^{\bar{\zeta}}$. Let $t \rightarrow \alpha_{t}$ be a one parameter group of automorphisms of $G$ leaving $\zeta$ invariant (this is going to represent the free evolution, in the application below). We define $B_{\alpha m}(G)$ to be the subspace of $B(G)$ of those functions $F$ such that

$$
t \in \mathbb{R} \rightarrow F\left(g_{1} \alpha_{t}\left(g_{2}\right) g_{3}\right), \quad g_{i} \in G, \quad i=1,2,3 .
$$

is Lebesgue measurable. It is clear that $B, B_{1}$, and $B_{\alpha m}$ are invariant subspaces with respect to both $R^{\zeta}$ and $L^{\bar{\zeta}}$.

Given any bounded complex measure $\mu$ on $G$ we define on $B(G)$ the following operators

$$
\begin{aligned}
& H_{I}^{\#} \equiv \lambda \int_{G} d \mu(g) R^{\zeta}(g), \\
& H_{I}^{b} \equiv \bar{\lambda} \int_{G} d \bar{\mu}(g) L^{\bar{\zeta}}(g),
\end{aligned}
$$

where $\lambda$ is a complex parameter and $\bar{\mu}$ denotes the complex conjugate measure to $\mu$, i.e. $\bar{\mu}(f)=\overline{\mu(\bar{f})}$ for any function $f$. To the one parameter group $t \rightarrow \alpha_{t}$ there corresponds an isometric operator $U_{\alpha_{t}}^{0} \equiv U_{t}^{0}$ on $B(G)$, defined by $\left(U_{t}^{0} F\right)(h) \equiv F\left(\alpha_{t}^{-1}(h)\right)$, and we want to find in $B(G)$ two functions $F_{t}^{\#}(g)$ and $F_{t}^{b}(g)$ solutions of the differential equations

$$
i \frac{\partial}{\partial t} F_{t}^{\#}(g)=\left(U_{-t}^{0} H_{I}^{\#} U_{t}^{0} F_{t}^{\#}\right)(g), \quad g \in G,
$$

with the initial condition

$$
\lim _{t \downarrow 0} F_{t}^{\#}(g)=F_{0}(g)
$$

and similar equations for $F_{t}^{b}$.

In what follows we shall discuss $F_{t}^{\#}$, the considerations for $F_{t}^{b}$ being entirely similar. This Cauchy problem uniquely defines $F_{t}^{\#}$ as a one cocycle

$$
F_{t+s}^{\#}=U_{-t}^{0}\left(U_{t}^{0} F_{t}^{\#}\right)_{s}, \quad t, s \in \mathbb{R}, \quad \text { see }[8] \text {. }
$$


Note that $U_{t}^{0} F_{t+s}$ is the solution of (2.8) with $t$ replaced by $s$ and with initial condition $U_{t}^{0} F_{t+s} l_{s=0}=U_{t}^{0} F_{t}$. It is very useful to describe the solution $F_{t}^{\#}$ in a compact way with the help of a Feynman path integral using a Poisson measure $P_{\mu}$, along the lines of e.g. $[12,14,16]$. We recall here only that $P_{\mu}$ is the Poisson measure for a Poisson process running in time $t$ on $G$ with intensity measure the positive bounded measure $d \mu$ on $G$ i.e., for any $f$ bounded Borel on $[0, t] \times G$

$$
\int \exp \left(i \int_{[0, t] \times G} f(t, g) d N_{\omega}(t, g)\right) d P_{\mu}(\omega)=\exp \left(\int_{[0, t] \times G}\left(e^{i f(\tau, g)}-1\right) d \tau d \mu(g)\right),
$$

where $N$ is the counting measure on countable subsets of $[0, t] \times G$. A realisation of $P_{\mu}(\omega)$ is as follows. Let $\Omega$ be the disjoint union of $\Omega_{n}$, where $n$ runs over the integers $\{0\} \cup \mathbb{N}, \Omega_{0}$ being an arbitrary one element set $\Omega_{0}=\left\{\omega_{0}\right\}$, and $\Omega_{n}$ for $n \in \mathbb{N}$ being the set of all $n$-tuples $\omega$ of the form

$$
\omega=\left\{\left(n, t_{1}, g_{1}\right), \ldots,\left(n, t_{n}, g_{n}\right)\right\}, \text { with } 0<t_{1}<\ldots<t_{n}<t, \quad g_{i} \in G, \quad i \in \mathbb{N} .
$$

Let $a_{i}$ be Lebesgue measurable subsets of $[0, t]$ with $a_{i} \cap a_{j}=\emptyset, B_{i}$ be Borel subsets of $G, i \in \mathbb{N}$, and define

$$
V_{a_{l}, B_{l}}^{(n)}=\left\{\omega \in \Omega_{n} \mid \omega=\left\{\left(n, t_{i}, g_{i}\right), 0<t_{1} \ldots<t_{n}<t, t_{i} \in a_{i}, g_{i} \in B_{i}, i=1, \ldots, n\right\} .\right.
$$

For any positive bounded measure $\mu$ on $G, P_{\mu}$ is then characterized by

$$
P_{\mu}\left(\Omega_{0}\right)=1, \quad P_{\mu}\left(V_{a_{i}, B_{\imath}}^{(n)}\right)=\prod_{i=1}^{n}\left|a_{i}\right| \mu\left(B_{i}\right),
$$

where $\left|a_{i}\right|$ is the Lebesgue measure of $a_{i}$. Equivalently, for any $F \in L^{1}\left(P_{\mu}\right)$, we have the following interpretation of above formula (2.11):

$$
\begin{aligned}
\int_{\Omega} F(\omega) d P_{\mu}(\omega)= & \sum_{n \geqq 0} \int_{0}^{t} d t_{n}, \ldots, \int_{0}^{t} d t_{1} \int_{G} d \mu\left(g_{n}\right) \ldots \int_{G} d \mu\left(g_{1}\right) \\
& \cdot F\left(\left\{\left(n, t_{1}, g_{1}\right), \ldots,\left(n, t_{n}, g_{n}\right)\right\}\right) .
\end{aligned}
$$

We refer to the papers quoted above for more details. Thus we have the theorem:

Theorem 2.1. Let $\mu$ be a complex measure on a topological group $G$. The solution of the evolution equation (2.8), with initial condition $F_{0} \in B_{\alpha m}(G)$ and with $H_{I}$ given by (2.6) is given by

$$
F_{t}^{\#}(g)=\int P_{|\lambda \mu|}(d \omega) \exp \{i \Phi(\omega)\} \exp \left\{i S_{0}^{r}(\omega)\right\} F_{0}\left(g G_{0}^{r}(\omega)\right),
$$

and similarly the solution of the evolution equation (2.8) with initial condition $F_{0} \in B_{\alpha m}(G)$ is given by

$$
F_{t}^{b}(g)=\int P_{\left|\lambda \mu^{*}\right|}(d \omega) \exp \left\{-i \Phi^{*}(\omega)\right\} \exp \left\{i S_{0}^{a}(\omega)\right\} F_{0}\left(G_{0}^{a}(\omega) g\right)
$$

where

$$
\exp \left\{i \Phi\left(n, t_{i}, g_{i}\right)\right\} \equiv(-i \lambda /|\lambda|)^{n} \exp \left\{i \sum_{j=1}^{n} \phi\left(g_{j}\right)\right\},
$$

$\phi$ being defined by

$$
d \mu(g)=\exp (i \phi(g)) d|\mu|(g) .
$$


Moreover

and

$$
\begin{aligned}
\exp \left\{i S_{0}^{r}\left(n, t_{i}, g_{i}\right)\right\} \equiv & \prod_{k=2}^{n} \zeta\left(\alpha_{-t_{n}}\left(g_{n}\right) \ldots \alpha_{-t_{k}}\left(g_{k}\right), \alpha_{-t_{k-1}}\left(g_{k-1}\right)\right) \\
& \cdot \zeta\left(g, \alpha_{-t_{n}}\left(g_{n}\right) \ldots \alpha_{-t_{1}}\left(g_{1}\right)\right)
\end{aligned}
$$

$$
G_{0}^{r}\left(n, t_{i}, g_{i}\right) \equiv \alpha_{-t_{n}}\left(g_{n}\right) \ldots \alpha_{-t_{1}}\left(g_{1}\right) \text {, }
$$

whereas

and

$$
\begin{gathered}
d \mu^{*}(g)=d \bar{\mu}\left(g^{-1}\right) \\
\exp \left\{i S_{0}^{a}\left(n, t_{i}, g_{i}\right)\right\}=\prod_{k=1}^{n-1} \zeta\left(\alpha_{-t_{k}}\left(g_{k}\right), \alpha_{-t_{k+1}}\left(g_{k+1}\right) \ldots \alpha_{-t_{n}}\left(g_{n}\right)\right) \\
\cdot \zeta\left(\alpha_{-t_{1}}\left(g_{1}\right) \ldots \alpha_{-t_{n}}\left(g_{n}\right), g\right)
\end{gathered}
$$

$$
G_{0}^{a}\left(n, t_{i}, g_{i}\right) \equiv \alpha_{-t_{1}}\left(g_{1}\right) \ldots \alpha_{-t_{n}}\left(g_{n}\right) .
$$

The solutions are entire functions of $\lambda$. [The suffixes $r$ and a stand for retarded (respectively, advanced) ].

Corollary 2.2. Let $\Omega_{t}^{\#}$ be defined by

$$
\left(\Omega_{t}^{\#} F_{0}\right)(g) \equiv F_{t}^{\#}(g),
$$

where $F_{t}^{\#}$ is the solution of (2.8) given by Theorem 2.1. Then $\Omega_{t}^{\#}$ is a linear map of $B_{\alpha m}(G)$ into itself, with norm bounded as follows

$$
\left\|\Omega_{t}^{\#}\right\| \leqq \exp \{t|\lambda||\mu|(G)\} .
$$

Define correspondly $\Omega_{t}^{b}$ by $\Omega_{t}^{b} F_{0} \equiv F_{t}^{b}$, then $\Omega_{t}^{b} \Omega_{t^{\prime}}^{\#}=\Omega_{t^{\prime}}^{\#} \Omega_{t}^{b}$ for all $t$ and $t^{\prime}$. One has

$$
\Omega_{t+s}=U_{t}^{0} \Omega_{s} U_{t}^{0} \Omega_{t} .
$$

and $U_{t}^{\#} \equiv U_{t}^{0} \Omega_{t}^{\#}, U_{t}^{b} \equiv U_{t}^{0} \Omega_{t}^{b}$ are groups of bounded operators on $B_{\alpha m}(G), t \in \mathbb{R}$.

Proof. For the bounds one uses the boundedness of $H_{I}^{\#}$. The group property follows easily from the cocyle relation (2.10).

We make now a remark concerning the ordering problem for Weyl quantization.

Lemma 2.3. Let $\zeta$ and $\xi$ be two equivalent multipliers on $G$, i.e. such that there exists a function $\lambda$ from $G$ to the one dimensional torus such that:

$$
\zeta\left(g_{1}, g_{2}\right)=\lambda\left(g_{1}\right) \lambda\left(g_{2}\right) \bar{\lambda}\left(g_{1} g_{2}\right) \xi\left(g_{1}, g_{2}\right), \quad g_{1}, g_{2} \in G .
$$

Then we have

$$
\begin{aligned}
\prod_{k=2}^{n} \zeta\left(\alpha_{-t_{n}}\left(g_{n}\right) \ldots \alpha_{-t_{k}}\left(g_{k}\right), \alpha_{-t_{k-1}}\left(g_{k-1}\right)\right) \zeta\left(\alpha_{-t}(g), \alpha_{-t_{n}}\left(g_{n}\right) \ldots \alpha_{-t_{1}}\left(g_{1}\right)\right) \\
=\prod_{k=2}^{n} \xi\left(\alpha_{-t_{n}}\left(g_{n}\right) \ldots \alpha_{-t_{k}}\left(g_{k}\right), \alpha_{-t_{k-1}}\left(g_{k-1}\right)\right) \xi\left(\alpha_{-t}(g), \alpha_{-t_{n}}\left(g_{n}\right) \ldots \bar{\alpha}_{-t_{1}}\left(g_{1}\right)\right) \\
\quad \cdot \prod_{\ell=1}^{n} \lambda\left(\alpha_{-t_{\ell}}\left(g_{\ell}\right)\right) \lambda\left(\alpha_{-t}(g)\right) \bar{\lambda}\left(\bar{\alpha}_{-t}(g) \bar{\alpha}_{-t_{n}}\left(g_{n}\right) \ldots \bar{\alpha}_{-t_{1}}\left(g_{1}\right)\right) .
\end{aligned}
$$


Remark. It follows from this that the functions $S_{0}^{r}, S_{0}^{a}$ defined in Theorem 2.1 depend trivially on $\zeta$ within a cohomology class. The following lemma is immediate

Lemma 2.4. If $\zeta$ is an $\alpha_{t}$-invariant bicharacter of G, i.e.

$$
\zeta\left(g_{1}, g_{2} \cdot g_{3}\right)=\zeta\left(g_{1}, g_{2}\right) \zeta\left(g_{2}, g_{3}\right), \quad g_{1}, g_{2}, g_{3} \in G,
$$

and

$$
\zeta\left(g_{1} \cdot g_{2}, g_{3}\right)=\zeta\left(g_{1}, g_{2}\right) \zeta\left(g_{2}, g_{3}\right)
$$

then

$$
\begin{aligned}
& \prod_{k=2}^{n} \zeta\left(\alpha_{-t_{n}}\left(g_{n}\right) \ldots \alpha_{-t_{k}}\left(g_{k}\right), \alpha_{-t_{k-1}}\left(g_{k-1}\right)\right) \\
& \quad=\prod_{i>j} \zeta\left(g_{i}, \alpha_{t_{t}-t_{j}}\left(g_{j}\right)\right) .
\end{aligned}
$$

Remark. Let $g \rightarrow W^{\zeta}(g)$ be a unitary projective representation of the canonical commutation relations on a Hilbert space $\mathscr{H}$, where $t \rightarrow \alpha_{1}$ is unitarily implemented by a strongly continuous unitary group $t \rightarrow \exp \left(i H_{0} t\right)$. Let $H_{I}$ be the bounded operator on $\mathscr{H}$ given by

$$
H_{I}=\lambda \int_{G} d v(g) W^{\zeta}(g)
$$

with $v$ a complex bounded measure on $G$. Then for any $\Phi, \Psi \in \mathscr{H}$, with $F_{\Phi, \Psi}^{W \zeta}$ defined by (2.5),

$$
\left(\Omega_{t}^{\#} F_{\Phi, \Psi}^{W \zeta}\right)(g)=\left(\Phi, W^{\zeta}(g) \exp \left(i t H_{0}\right) \exp \left(-i\left(H_{0}+H_{I}\right) t\right) \Psi\right),
$$

is not a group operation with respect to $t$, but a one cocyle. On the other hand

$$
\left(U_{t}^{\#} F_{\Phi, \Psi}^{W \zeta}\right)(g)=\left(\Phi, \exp \left(i t H_{0}\right) W^{\zeta}(g) \exp \left(-i\left(H_{0}+H_{I}\right) t\right) \Psi\right)
$$

is a group operation with respect to $t$. Similar formulae hold for $\Omega_{t}^{b}$ and $U_{t}^{b}$.

Now we are in position to state a theorem which gives the perturbed free evolution in the Schrödinger picture [see formula (1.1) in the introduction]. We call $\Sigma$ the cone consisting of functions $F \in B_{\alpha m}(G)$ of $\zeta$-positive type, i.e. such that

$$
\sum_{i, j=1}^{N} \lambda_{i} \bar{\lambda}_{j} \bar{\zeta}\left(g_{i} g_{j}^{-1}, g_{i}\right) F\left(g_{i} g_{j}^{-1}\right) \geqq 0
$$

for any choice of $\lambda_{i} \in \mathbb{C}, g_{i} \in G, i, j=1, \ldots, N, N \in \mathbb{N}$.

Theorem 2.5. Let $\alpha_{t}^{H}$ be defined by

$$
\alpha_{t}^{H} \equiv U_{t}^{0} \Omega_{t}^{\#} \Omega_{t}^{b},
$$

where $U_{t}^{0}(h) \equiv F\left(\alpha_{t}^{-1}(h)\right), \quad F \in B(G), \alpha_{t}$ being a one-parameter automorphism and $\Omega_{t}^{\#}, \Omega_{t}^{b}$ being defined in Corollary 2.2. Then $\alpha_{t}^{H}$ is a one parameter continuous subgroup of bounded operators on $B_{\alpha m}(G)$ leaving invariant the cone $\Sigma$.

Proof. The proof follows easily from Theorem 2.1 and Corollary 2.2. 
Remark. $\alpha_{t}^{H}$ can be explicitly computed. We give its expression in the most useful case where $\lambda$ is real and $\mu=\mu^{*}$, which corresponds to a real potential

$$
\begin{aligned}
\left(\alpha_{t}^{H} F\right)(g)= & \sum_{m, n \geqq 0} \lambda^{m+n}(-i)^{m+n} \int_{0}^{t} d s_{m} \ldots \int_{0}^{s_{2}} d s_{1} \int_{0}^{t} d t_{n} \ldots \int_{0}^{t_{2}} d t_{1} \\
& \cdot \int_{G} d \mu\left(g_{m}\right) \ldots \int_{G} d \mu\left(g_{1}\right) \int_{G} d \mu\left(h_{n}\right) \ldots \int_{G} d \mu\left(h_{1}\right) \\
& \cdot \prod_{k=1}^{m-1} \zeta\left(\alpha_{-s_{1}}\left(g_{1}\right) \ldots \alpha_{-s_{k}}\left(g_{k}\right), \alpha_{-s_{k-1}}\left(g_{k-1}\right)\right) \\
& \cdot \prod_{\ell=1}^{n-1} \zeta\left(\alpha_{-t_{\ell+1}}\left(h_{\ell+1}\right), \alpha_{-t_{\ell}}\left(h_{\ell}\right) \ldots \alpha_{-t_{1}}\left(h_{1}\right)\right) \\
& \cdot \zeta\left(\alpha_{-s_{1}}\left(g_{1}\right) \ldots \alpha_{-s_{m}}\left(g_{m}\right), \alpha_{-t}(g)\right) \\
& \cdot \zeta\left(\alpha_{-s_{1}}\left(g_{1}\right) \ldots \alpha_{-s_{m}}\left(g_{m}\right) \alpha_{-t}(g), \alpha_{-t_{n}}\left(h_{n}\right) \ldots \alpha_{-t_{1}}\left(h_{1}\right)\right. \\
& \cdot F\left(\alpha_{-s_{1}}\left(g_{1}\right) \ldots \alpha_{-s_{m}}\left(g_{m}\right) \alpha_{-t}(g) \alpha_{-t_{n}}\left(h_{n}\right) \ldots \alpha_{-t_{1}}\left(h_{1}\right)\right) .
\end{aligned}
$$

The next proposition gives a Schrödinger equation for $\alpha_{t}^{H}$, in the sense that it gives an affine, one parameter group of transformations of the set of states. This equation will enter as an essential tool in Sect. 4 to prove the existence of a thermodynamical limit for relativistic quantum field models. Let $H_{0}$ be the infinitesimal generator of $U_{t}^{0}$ defined on a dense domain $D\left(H_{0}\right)$ in $B_{\alpha m}(G)$.

Theorem 2.6. If $G$ is an abelian group, for any $F$ in $D\left(H_{0}\right)$ the following equation holds:

$$
i \frac{\partial}{\partial t}\left(\alpha_{t}^{H} F\right)(g)=\left(H_{0} \alpha_{t}^{H} F\right)(g)+\lambda \int_{G} d \mu(h)(\zeta(g, h)-\zeta(h, g))\left(\alpha_{t}^{H} F\right)(g h) .
$$

Proof. This follows easily from Theorem 2.1 .

\section{Field Theory with Trigonometric Interaction and Cut-Offs}

This section is an application of the previous results to a model for quantum field theory, the trigonometric interaction in $s+1$ dimensional space time, with ultraviolet and space cut-offs.

Let $\mathscr{S}_{R}\left(\mathbb{R}^{s}\right) \equiv \mathscr{S}_{R}$ (respectively, $\mathscr{D}_{R}\left(\mathbb{R}^{s}\right) \equiv \mathscr{D}_{R}$ ) be the Schwartz space of real $C^{\infty}$-functions of rapid decrease (respectively with compact support). They are abelian groups under pointwise addition. We look at:

$$
G=\mathscr{S}_{R} \times \mathscr{S}_{R}, \quad\left(\text { respectively, } \mathscr{D}_{R} \times \mathscr{D}_{R}\right),
$$

as the space of initial conditions of a relativistic neutral scalar Bose field in $s+1$ space-time dimensions.

Corresponding to the equal time commutation relations of such a field there is a central extension of $G$ which is given, choosing units such that $\hbar=1$, by a multiplier $\zeta$, where 


$$
\zeta\left((f, g),\left(f^{\prime}, g^{\prime}\right)\right)=\exp \left\{\frac{i}{2} \int_{\mathbb{R}^{s}} d x\left\{f^{\prime}(x) g(x)-f(x) g^{\prime}(x)\right\}\right\},
$$

for all $(f, g),\left(f^{\prime}, g^{\prime}\right) \in G$.

Consider a projective representation of the C.C.R. in a Hilbert space $\mathscr{H}$ with multiplier $\zeta$, and let $\phi(f)$ be the field operator (at time zero) and $\Pi(g)$ its canonical conjugate momentum with $f, g \in \mathscr{S}_{R}$. Provided that $\lambda \in \mathbb{R} \rightarrow W_{\lambda f, g}^{\zeta}$ and $\mu \in \mathbb{R} \rightarrow W_{f, \mu g}$ are continuous, the Weyl operators are defined as the unitary operators

on $\mathscr{H}$. We have:

$$
W_{g, f}^{\zeta}=\exp \{i(\Pi(g)-\phi(f))\},
$$

$$
W_{g, f^{\prime}}^{\zeta} W_{g^{\prime}, f^{\prime}}^{\zeta}=\zeta\left((f, g),\left(f^{\prime}, g^{\prime}\right)\right) W_{g+g^{\prime}, f+f^{\prime}}^{\zeta},
$$

for all $(f, g),\left(f^{\prime}, g^{\prime}\right) \in G$. For all $\Phi$ in $\mathscr{H}$ the map

$$
(f, g) \in G \rightarrow\left(\Phi, W_{g, f}^{\zeta} \Phi\right)
$$

belongs to the space $B(G)$ of bounded complex functionals on $G$.

As discussed in Sect. 2, $B(G)$ carries two isometric projective representations $R^{\zeta}$ and $L^{\bar{\zeta}}$ of $G$ given by (2.2), i.e.

$$
\begin{aligned}
& \left\{R^{\zeta}(f, g) F\right\}\left(h_{1}, h_{2}\right)=\exp \left\{\frac{i}{2} \int_{\mathbb{R}^{s}} d x\left\{f(x) h_{2}(x)-g(x) h_{1}(x)\right\}\right\} F\left(f+h_{1}, g+h_{2}\right), \\
& \left\{L^{\bar{\zeta}}(f, g) F\right\}\left(h_{1}, h_{2}\right)=\exp \left\{\frac{i}{2} \int_{\mathbb{R}^{s}} d x\left\{f(x) h_{2}(x)-g(x) h_{1}(x)\right\}\right\} F\left(h_{1}-f, h_{2}-g\right),
\end{aligned}
$$

for all $f, g, h_{1}, h_{2} \in \mathscr{S}_{R}\left(\mathbb{R}^{s}\right)$.

Now we shall define a group of automorphisms $\alpha_{t}$ of $G$, leaving $\zeta$ invariant, and consider as in Sect. 2 the fourtuple $\left(B, \alpha_{t}, R^{\zeta}, L^{\bar{\zeta}}\right) . \alpha_{t}$ corresponds to the free field time evolution, usually defined for free quantum fields in Fock space. We define $\alpha_{t}$ as the map of $G$ in itself given by

$$
\alpha_{t}(f, g)=\left(f_{t}, g_{t}\right)
$$

where $f_{t}$ and $g_{t}$ are defined by their Fourier transform as follows:

$$
\begin{gathered}
\tilde{f}_{t}(p)=\cos (\omega(p) t) \tilde{f}(p)+\omega(p)^{-1} \sin (\omega(p) t) \tilde{g}(p), \\
\tilde{g}_{t}(p)=\partial_{t} \tilde{f}_{t}(p),
\end{gathered}
$$

with $\omega(p) \equiv \sqrt{p^{2}+m^{2}}$. Thus for $t>0$,

$$
\begin{gathered}
f_{t}(x)=\left(\partial_{t} \Delta_{r}^{m} * f\right)(t, x)+\left(\Delta_{r}^{m} * g\right)(t, x), \\
g_{t}(x)=\partial_{t} f_{t}(x),
\end{gathered}
$$

where $\Delta_{r}^{m}$ is the retarded relativistic propagator:

with

$$
\begin{gathered}
\Delta_{\boldsymbol{r}}^{m}(t, x)=\frac{\theta(t)}{(2 \pi)^{s}} \int_{\mathbb{R}^{s}} \frac{d p}{\omega(p)} \sin (\omega(p) t) e^{i p x}, \\
\theta(t)=\left\{\begin{array}{lll}
0 & \text { if } & t<0 \\
1 & \text { if } & t>0 .
\end{array}\right.
\end{gathered}
$$


$\alpha_{t}$ extends to a group of isometric transformations $U_{\alpha_{t}}^{0}$ of $B(G)$, defined by:

$$
\left\{U_{\alpha_{t}}^{0} F\right\}(f, g)=F\left(\alpha_{t}^{-1}(f, g)\right) .
$$

$U_{\alpha_{t}}^{0}$ is continuous and isometric on the Banach space $B_{\alpha m}(G)$.

Also $B_{\alpha m}(G)$ carries an isometric representation of the Poincaré group in $\mathbb{R}^{s+1}$, which leaves the multiplier $\zeta$ invariant. The infinitesimal generators of the Poincaré group are given in [7].

Now we want to give the corresponding representation for interacting fields with trigonometric interaction. This type of interaction has been studied, e.g. in [30]. They are defined in a bounded domain $\Lambda$ of $\mathbb{R}^{s}$, which we take for convenience to be a symmetric cubic box,

$$
\Lambda=\left\{x \in \mathbb{R}^{s} ;\left|x_{i}\right|<a, i=1,2, \ldots, s ; a>0\right\},
$$

by a Hamiltonian $H^{4 \kappa}=H_{0}+V$ with:

$$
V=\lambda \int_{\Lambda} d x \int_{\mathbb{R}} d v(\alpha) \exp \left\{-i \alpha \phi_{\kappa}(x)\right\},
$$

defined in the Fock representation of the C.C.R. Now $H_{0}$ is the free relativistic Hamiltonian and $\phi_{\kappa}(x)=\left(\phi * \chi_{\kappa}\right)(x)$ is the time zero free field regularized by an ultraviolet cut-off function $\chi_{\kappa}(x)=\kappa^{s} \chi(\kappa x), \kappa>0$, where $\chi$ is a positive symmetric $C^{\infty}$-function with support in the unit ball of $\mathbb{R}^{s}$ and such that $\int_{\mathbb{R}^{s}} \chi(x) d x=1$. Here $v$ is a complex measure on $\mathbb{R}$ and $\lambda$ is a positive constant. As in Sect. 2 we denote by $\phi$ the phase which appears in the polar decomposition of $v$ :

$$
d v(\alpha)=\exp \{i \phi(\alpha)\} d|v|(\alpha) .
$$

In order to have a self-adjoint Hamiltonian, we require that

$$
\bar{v}(\alpha)=v(-\alpha) \text {. }
$$

We remark that (3.19) implies that (3.17) can be written as

$$
V=2 \lambda \int_{A} d x \int_{0}^{\infty} d|v|(\alpha) \cos \left(\alpha \phi_{\kappa}(x)-\phi(\alpha)\right) .
$$

A particular case of interaction of the form (3.17) is the sine-Gordon model, see e.g. [22], which corresponds to the Bernoulli measure:

$$
d v(\alpha)=\frac{1}{2}\left\{\delta_{\alpha_{0}}+\delta_{-\alpha_{0}}\right\} .
$$

To define the Poisson measure entering in Theorem 2.1 in our present case, we shall rewrite (3.17) in term of the Weyl operators $W_{f_{1} f_{2}}^{\zeta}, f_{i} \in \mathscr{S}_{R}\left(\mathbb{R}^{s}\right)$ associated with the Fock representation of the C.C.R. We have for the interaction $V$ with ultraviolet and space cut-offs

$$
V=\lambda \int_{\Lambda} d x \int_{\mathbb{R}} d v(\alpha) W_{0, \alpha \chi_{\kappa}^{x}}^{\mathrm{Fock}}
$$

with $\chi^{x}(y) \equiv \chi(x-y)$. Here $W_{f, g}^{\text {Fock }}$ stands for the Weyl operator in the Fock representation, with the usual multiplier given by (3.2). 
For any two vectors $\Phi$ and $\Psi$ in the Fock representation we then get:

$$
\left(\Phi, W_{f, g}^{\text {Fock }} V \Psi\right)=\left(V^{\#} F_{\Phi, \Psi}^{\text {Fock }}\right)(f, g), \quad\left(\Phi, V W_{f, g}^{\text {Fock }} \Psi\right)=\left(V^{b} F_{\Phi, \Psi}^{\text {Fock }}\right)(f, g),
$$

where $F_{\Phi, \Psi}^{\text {Fock }}(f, g) \equiv\left(\Phi, W_{f, g}^{\text {Fock }} \Psi\right), V^{\#} \equiv \lambda \int_{\Lambda} d x \int_{\mathbb{R}} d v(\alpha) R_{0, \alpha \chi_{\kappa}^{\varkappa}}^{\zeta}$, and similarly for $V^{b}$, with $R^{\zeta}$ replaced by $L^{\bar{\zeta}}$.

Furthermore $\alpha_{t}$ [from (3.8)], is unitarily implemented in the Fock representation by the weakly continuous unitary group $U_{t}^{0}=\exp \left(-i H_{0} t\right)$. We have the perturbation series:

$$
\begin{aligned}
\left(\Phi \mid W_{f, g}^{\text {Fock }} \exp \left\{-i t H_{0}\right\} \exp \left\{i t\left(H_{0}+V\right)\right\} \Psi\right) \\
=\sum_{n \geqq 0}(i \lambda)^{n} \int_{0}^{t} d t_{n} \ldots \int_{0}^{t_{2}} d t_{1}\left(\Phi \mid W_{f, g}^{\text {Fock }} V_{t_{n}} \ldots V_{t_{1}} \Psi\right) \\
=\sum_{n \geqq 0}(i \lambda)^{n} \int_{0}^{t} d t_{n} \ldots \int_{0}^{t_{2}} d t_{1} \int_{G} d \varrho\left(f_{n}, g_{n}\right) \ldots \int_{G} d \varrho\left(f_{1}, g_{1}\right) \\
\quad \cdot\left\{R_{\left(f_{n}, g_{n}\right) t_{n}}^{\zeta} \ldots R_{\left(f_{1}, g_{1}\right)_{1}}^{\zeta} F_{\Phi, \Psi}^{\text {Fock }}\right\}(f, g),
\end{aligned}
$$

where $V(t) \equiv U_{t}^{0} V U_{-t}^{0},(f, g)_{t} \equiv \alpha_{t}(f, g) \equiv\left(f_{t}, g_{t}\right)[\mathrm{cf}$. (3.8)], and

$$
d \varrho(f, g)=\int_{\Lambda} d x \int_{\mathbb{R}} d v(\alpha) \delta_{0}^{(f)} \delta_{\alpha \chi_{\kappa}^{2}}(g)
$$

the $\delta$ being the evaluation measures on $\mathscr{S}_{R}\left(\mathbb{R}^{s}\right)$. A similar formula holds for the matrix elements $\left(\Phi, \exp \left(-i\left(H_{0}+V\right) \exp \left(i H_{0} t\right) W_{f, g}^{\text {Fock }} \Psi\right)\right.$. We remark that the operators $V^{\#}$ and $V^{b}$ in (3.22) can be written as

$$
V^{\#}=\lambda \int_{G} d \varrho(g) R_{g}^{\zeta} \quad\left[\text { respectively, } V^{b}=\lambda \int_{G} d \varrho(g) L_{g}^{\bar{\zeta}}\right],
$$

As in Sect. 2, Theorem 2.1, it is useful to express the solution of the Schrödinger equation associated with $H_{0}+V$, by a Poisson measure associated with the group $G$.

In the present situation we can also characterize this measure as the measure $\hat{P}_{\varrho}$ defined by

$$
\begin{aligned}
\int \exp \{i\langle f, \xi\rangle\} d \hat{P}_{\varrho}(\xi)= & \exp \left\{\lambda \int_{0}^{t} d \tau \int_{\Lambda} d x \int_{\mathbb{R}} d|v|(\alpha)\right. \\
& \left.\cdot\left(\exp \left(-i \alpha \int_{0}^{\tau} d \sigma \iint d u d v f(\sigma, u) \Delta_{r}^{m}(\tau-\sigma, u-v) \chi_{\kappa}^{x}(v)\right)-1\right)\right\},
\end{aligned}
$$

the integration on the left hand side being over $\mathscr{S}_{R}^{\prime}\left(\mathbb{R}^{s}\right) \times \mathscr{S}_{R}^{\prime}\left(\mathbb{R}^{s}\right) \times C_{0}^{\infty}\left([0, t]^{\prime}\right)$, where

$$
f \in \mathscr{S}_{R}\left(\mathbb{R}^{s}\right) \times \mathscr{S}_{R}\left(\mathbb{R}^{s}\right) \times C_{0}^{\infty}(0, t),
$$

hence $f$ is indefinitely differentiable and of compact support with respect to the variable $\tau$. In fact $\hat{P}_{\varrho}$ has support on elements of

$$
\mathscr{S}_{R}^{\prime}\left(\mathbb{R}^{s}\right) \times \mathscr{S}_{R}^{\prime}\left(\mathbb{R}^{s}\right) \times C_{0}^{\infty}(0, t)^{\prime}
$$


of the form: $\left(\delta_{0}, \delta_{\alpha \chi_{\kappa}^{x}}, \delta_{t^{\prime}}\right)$, where $\alpha \in \operatorname{Support}(v)$ and $t^{\prime} \in[0, t]$. Let $P_{\mu}$ be the measure image of $\hat{P}_{\varrho}$ under the transformation of $\mathscr{S}_{R}^{\prime} \times \mathscr{S}_{R}^{\prime} \times \mathrm{C}_{0}^{\infty}(0, \mathrm{t})^{\prime}$ into $(0, t) \times \Lambda \times \mathbb{R}$ induced by the inverse of the mapping $(s, x, \alpha) \rightarrow \alpha \chi_{\kappa}^{x} \otimes \delta_{s}$. Then $P_{\mu}$ is the measure introduced in Sect. 2, Theorem 2.1, with $G$ replaced by $\Lambda \times \mathbb{R}$ and $d \mu$ by $d x \times d v(\alpha)$. Since $P_{\mu}$ depends on $\Lambda, t, \lambda|v|$ we shall denote it from now on by $P_{\lambda|v|}^{\Lambda, t}$.

Let us recall the essential definitions needed to state the basic result of this section. Let $U_{t}^{0} \equiv U_{\alpha_{t}}^{0}$ be the isometric operator given by the free evolution $\alpha_{t}$ defined by (3.8). Let $\Omega_{t}^{\#}$ be the linear bounded operator on $B_{\alpha m}(G)$ given, corresponding to Theorem 2.1 , by

$$
\left(\Omega_{t}^{\#} f\right)(g)=\int P_{\lambda|v|}^{\Lambda, t}(d \omega) \exp \left\{i \Phi(\omega)+i S_{0}^{r}(\omega)\right\} F\left(g+G_{0}^{r}(\omega)\right),
$$

where $g \in G ; F \in B_{\alpha m}(G)$,

$$
\exp \left\{i \Phi\left(n, t_{j} ; x_{j} ; \alpha_{j}\right\}=\exp \left(-i \frac{\pi}{2} n+i \sum_{j=1}^{n} \phi\left(\alpha_{j}\right)\right),\right.
$$

with $\phi$ given by (3.18) and

$$
\begin{aligned}
\exp \left\{i S_{0}^{r}\left(n, t_{i} ; x_{i}, \alpha_{i}\right)\right\}= & \prod_{\kappa=2}^{n} \zeta\left(\alpha_{-t_{n}}\left(g_{n}\right)+\ldots+\alpha_{-t_{\kappa}}\left(g_{\kappa}\right), \alpha_{-t_{\kappa-1}}\left(g_{\kappa-1}\right)\right) \\
& \zeta\left(g, \alpha_{-t_{n}}\left(g_{n}\right)+\ldots+\alpha_{-t_{1}}\left(g_{1}\right)\right),
\end{aligned}
$$

where $\zeta$ is the multiplier defined in (3.2), and $g_{i}=\left(0, \alpha_{i} \chi_{\kappa}^{x_{0}}\right)$. Moreover

$$
G_{0}^{r}\left(n, t_{i}, x_{i}, \alpha_{i}\right)=\alpha_{-t_{n}}\left(g_{n}\right)+\ldots+\alpha_{-t_{1}}\left(g_{1}\right) .
$$

Correspondingly as in Sect. 2, we have a similar expression for $\Omega_{t}^{b}$.

Finally we recall the definition of the automorphism group $\alpha_{t}^{H}$ of Sect. 2, where here $H=H^{\Lambda, \kappa}=H_{0}+V$ :

$$
\alpha_{t}^{H}=U_{t}^{0} \Omega_{t}^{\#} \Omega_{t}^{b},
$$

with the right hand side defined correspondingly to Theorem 2.5. We have the following theorem:

Theorem 3.1. The automorphism group $\alpha_{t}^{H}$ associated with the Hamiltonian $H^{\Lambda, \kappa}$ with trigonometric interaction $V$ given by (3.17) maps linearly and boundedly $B_{\alpha m}\left(\mathscr{S}_{R}\left(\mathbb{R}^{s}\right) \times \mathscr{S}_{R}\left(\mathbb{R}^{s}\right)\right)$ into itself and gives a solution of the Schrödinger equation on the Banach space $B_{x m}\left(\mathscr{S}_{R}\left(\mathbb{R}^{s}\right) \times \mathscr{S}_{R}\left(\mathbb{R}^{s}\right)\right)$ :

$$
i \frac{\partial}{\partial t}\left(\alpha_{t}^{H} F\right)(g)=H_{0} \alpha_{t}^{H} F(g)+\lambda \int_{G} d \varrho(g)\{\zeta(g, h)-\zeta(h, g)\} \alpha_{t}^{H} F(g+h),
$$

with initial conditions $F$ in

$$
B_{\alpha m}\left(\mathscr{S}_{R}\left(\mathbb{R}^{s}\right) \times \mathscr{S}_{R}\left(\mathbb{R}^{s}\right)\right) \cap C^{1}\left(\mathscr{S}_{R}\left(\mathbb{R}^{s}\right) \times \mathscr{S}_{R}\left(\mathbb{R}^{s}\right)\right) .
$$

We have:

$$
\alpha_{t}^{H} F\left(h_{1}, h_{2}\right)=\int P_{\lambda|v|}^{\Lambda, t}\left(d \omega_{1}\right) P_{\lambda|v|}^{\Lambda, t}\left(d \omega_{2}\right) \exp \left\{i \Phi\left(\omega_{1}, \omega_{2}\right)\right\} F_{\kappa, t}\left(h_{1}, h_{2}\right)\left(\omega_{1}, \omega_{2}\right),
$$


where writing $\left(m, s_{i}, x_{i}, \alpha_{i}\right)$ for $\omega_{1}$ and $\left(n, t_{j}, y_{j}, \beta_{j}\right)$ for $\omega_{2}$ :

$$
\begin{aligned}
F_{\kappa, t}\left(h_{1}, h_{2}\right)\left(\left(m, s_{i}, x_{i}, \alpha_{i}\right),\left(n, t_{j}, y_{j}, \beta_{j}\right)\right) \\
=\exp \left\{\frac{i}{2} \sum_{i, i^{\prime}} \alpha_{i} \alpha_{i^{\prime}}\left(\chi_{\kappa} * \Delta_{a}^{m} * \chi_{\kappa}\right)\left(s_{i}-s_{i^{\prime}}, x_{i}-x_{i^{\prime}}\right)\right\} \\
\cdot \cdot \exp \left\{\frac{i}{2} \sum_{j, j^{\prime}} \beta_{j} \beta_{j^{\prime}}\left(\chi_{k} * \Delta_{r}^{m} * \chi_{\kappa}\right)\left(t_{j}-t_{j^{\prime}}, y_{j}-y_{j^{\prime}}\right)\right\} \\
\cdot \exp \left\{\frac{i}{2} \sum_{i, j} \alpha_{i} \beta_{j}\left(\chi_{k} * \Delta^{m} * \chi_{\kappa}\right)\left(s_{i}-t_{j} ; x_{i}-y_{j}\right)\right\} \\
\cdot \exp \left\{\frac{i}{2}\left(\sum_{i=1}^{m} \alpha_{i}\left(\left(\partial_{0} \chi_{k} * \Delta^{m} * h_{1}\right)\left(s_{i}-t, x_{i}\right)-\left(\chi_{\kappa} * \Delta^{m} * h_{2}\right)\left(s_{i}-t, x_{i}\right)\right)\right)\right\} \\
\cdot \exp \left\{-\frac{i}{2}\left(\sum_{j=1}^{n} \beta_{j}\left(\left(\partial_{0} \chi_{k} * \Delta^{m} * h_{1}\right)\left(t_{j}-t, y_{j}\right)-\left(\chi_{\kappa} * \Delta^{m} * h_{2}\right)\left(t_{j}-t, y_{j}\right)\right)\right)\right\} \\
\cdot F\left(h_{1,-t}+\sum_{i=1}^{m} \alpha_{i}\left(\partial_{0} \chi_{\kappa} * \Delta^{m}\right)\left(s_{i}, x_{i}\right)+\sum_{j=1}^{n} \beta_{j}\left(\partial_{0} \chi_{\kappa} * \Delta^{m}\right)\left(t_{j}, y_{j}\right),\right. \\
\left.\quad h_{2,-t}+\sum_{i=1}^{m} \alpha_{i}\left(\chi_{\kappa} * \Delta^{m}\right)\left(s_{i}, x_{i}\right)+\sum_{j=1}^{n} \beta_{j}\left(\chi_{\kappa} * \Delta^{m}\right)\left(t_{j}, y_{j}\right)\right),
\end{aligned}
$$

and the phase $\Phi\left(\omega_{1}, \omega_{2}\right)$ is equal to:

$$
\Phi\left(\left(m, s_{i}, x_{i}, \alpha_{i}\right),\left(n, t_{j}, y_{j}, \beta_{j}\right)\right)=\frac{\pi}{2}(m-n)+\sum_{i=1}^{m} \phi\left(\alpha_{i}\right)+\sum_{j=1}^{n} \phi\left(\beta_{j}\right),
$$

$h_{i,-t}$ are defined as in (3.9). Moreover $\Delta_{r}^{m}, \Delta_{a}^{m}$, and $\Delta^{m}$ are the invariant distributions given by (3.13) and respectively by

$$
\begin{aligned}
& \Delta_{a}^{m}(t, x) \equiv \frac{1-\theta(t)}{(2 \pi)^{s}} \int_{\mathbb{R}^{s}} d p e^{i p x} \sin (\omega(p) t) / \omega(p), \\
& \Delta^{m}(t, x) \equiv \frac{1}{(2 \pi)^{s}} \int_{\mathbb{R}^{s}} d p e^{i p x} \sin (\omega(p) t) / \omega(p) .
\end{aligned}
$$

Remark. In the case of nonrelativistic one dimensional quantum mechanics for a system with Hamiltonian $H$ given by

$$
H=-\frac{d^{2}}{d q^{2}}+q^{2}+V(q)
$$

with $V(q)=\int_{\mathbb{R}} \exp (-i \alpha q) d v(\alpha)$, the group $G$ is $\mathbb{R}^{2}$ and the corresponding Schrödinger equation can be written from Theorem 2.1 as

$$
i \frac{\partial}{\partial t} \alpha_{t} F(q, p)=H_{0} \alpha_{t} F(q, p)+2 i \int_{\mathbb{R}} d v(\alpha) \sin (-\alpha q / 2) \alpha_{t} F(q, p-\alpha)
$$

(cf. [33]). 


\section{Removal of the Cut-Offs}

As we shall see the removal of the cut-off $\kappa$ in the formulas of Theorem 3.1 is only possible on some but not all functionals $F$ on $\mathscr{S}_{R}\left(\mathbb{R}^{s}\right) \times \mathscr{S}_{R}\left(\mathbb{R}^{s}\right)$. For instance if $F$ is the well known vacuum functional associated with the Fock representation, then the argument of $F$ in the formulas of Theorem 3.1 become singular as $\kappa \rightarrow \infty$ for all $s \geqq 1$. For $s=1, m=0$, one can come over this difficulty by the usual renormalization procedure [16].

In this section, we shall prove that both limits $\kappa \rightarrow \infty$ and $\Lambda \uparrow \mathbb{R}^{s}$ exist for a large subset of functionals $F$. We consider first the functionals $E_{k_{1}, k_{2}}$ defined as follows, for any pairs $k_{1}, k_{2} \in \mathscr{S}_{R}^{\prime}\left(\mathbb{R}^{s}\right)$ and $h_{1}, h_{2} \in \mathscr{S}_{\mathbb{R}}\left(\mathbb{R}^{s}\right)$ :

$$
E_{k_{1}, k_{2}}\left(h_{1}, h_{2}\right) \equiv \exp \left\{\frac{i}{2} \int_{\mathbb{R}^{s}} d x\left\{h_{1}(x) k_{2}(x)-h_{2}(x) k_{1}(x)\right\},\right.
$$

where the integral on the right hand side is interpreted in the sense of distributions. Obviously one has:

$$
E_{k_{1}, k_{2}}(0,0)=E_{0,0}\left(h_{1}, h_{2}\right)=1 \text {, for all } k_{1}, k_{2} \in \mathscr{S}_{\boldsymbol{R}}^{\prime}\left(\mathbb{R}^{s}\right) \text {, and all } h_{1}, h_{2} \in \mathscr{S}_{\boldsymbol{R}}\left(\mathbb{R}^{s}\right) \text {. }
$$

Remark. These functionals are not states; however in some cases they can be used to express states. Let us first look at the case of nonrelativistic quantum mechanics with one degree of freedom. Here we have for instance the formula:

$$
(\Omega, \exp \{i(p Q-q P)\} \Omega)=\frac{1}{4 \pi} \int_{\mathbb{R}^{2}} d x \exp \left\{-\frac{1}{2}\|x\|^{2}+\frac{i}{2}\left(p x_{1}-q x_{2}\right)\right\},
$$

where $\Omega$ is the ground state of the harmonic oscillator. Since $\exp \left(\frac{i}{2}\left(p x_{1}-q x_{2}\right)\right)$ plays the role in this case of the functionals $E_{k_{1}, k_{2}}$, this formula shows that we can describe the fundamental state of quantum mechanics by a superposition of functionals of the form $E_{k_{1}, k_{2}}$.

This integral representation extends also to the Fock vacuum state of relativistic free fields (see e.g. [41]), hence in the free field case one recovers the vacuum from the functionals $E_{k_{1}, k_{2}}$.

We shall call $\mathscr{L}$ the linear subspace of $B\left(\mathscr{S}_{R}\left(\mathbb{R}^{s}\right) \times \mathscr{S}_{R}\left(\mathbb{R}^{s}\right)\right)$ consisting of all finite linear combinations of $E_{k_{1}, k_{2}}$, i.e.

$$
\mathscr{L} \equiv\left\{\sum_{i=1}^{N} \alpha_{i} E_{k_{1}^{i}, k_{2}^{i}}, \alpha_{i} \in \mathbb{C}, k_{1}^{i}, k_{2}^{i} \in \mathscr{S}_{R}^{\prime}\left(\mathbb{R}^{s}\right), i=1,2, \ldots, N\right\} .
$$

We remark that $\mathscr{L}$ is invariant under the action of the Poincare group associated with the free fields. Below we shall consider a subspace $\mathscr{L}_{0}$ of $\mathscr{L}$ defined as $\mathscr{L}$ with $k_{1}, k_{2}$ taken to be such that, in the sense of generalized functions, $\left\langle k_{j}^{i}, T\right\rangle$ are well defined, where $T$ is either $\Delta^{m}$ or $\partial_{0} \Delta^{m}$.

It is also interesting to introduce another subspace of bounded functionals on $B(G)$, namely the linear subspace $\mathfrak{M}$ consisting of finitely based functionals which are linear combinations of functionals $F_{H_{2 n}}^{f}$ described as follows: Let $\left\{\phi_{n}\right\}_{n \in \mathbb{N}}$ be a family of functions in $\mathscr{S}_{\mathbb{R}}\left(\mathbb{R}^{s}\right)$ which is separating in $L^{2}\left(\mathbb{R}^{s}, d x\right)$. Let $H_{2 n}$ be the subspace of $L_{R}^{2}\left(\mathbb{R}^{s}, d x\right)$ generated by $\left\{\phi_{i}\right\}_{i=1,2, \ldots, 2 n^{*}}$. Let $f$ be an arbitrary function 
on $\mathbb{R}^{2 n}$, which is the Fourier transform of a bounded complex measure on $\mathbb{R}^{2 n}$. The functional $F_{H_{2 n}}^{f}$ evaluated on $\left(h_{1}, h_{2}\right) \in \mathscr{S}_{R} \times \mathscr{S}_{R}$ is by definition:

$$
F_{H_{2 n}}^{f}\left(h_{1}, h_{2}\right) \equiv \begin{cases}f\left(\left(h_{1}, \phi_{1}\right),\left(h_{2}, \phi_{2}\right), \ldots,\left(h_{1}, \phi_{2 n-1}\right),\left(h_{2}, \phi_{2 n}\right)\right), & \text { if } h_{1}, h_{2} \in H_{2 n}, \\ 0 & \text { otherwise. }\end{cases}
$$

By definition $\mathfrak{M}$ is the complex linear space of the $F_{H_{2 n}}^{f}$ for all $n$ and all possible $f$. We remark that $F_{H_{2 n}}^{f}$ can be written in the form:

$$
F_{H_{2 n}}^{f}\left(h_{1}, h_{2}\right)=\int d \mu\left(k_{1}, k_{2}\right) \exp \left\{\frac{i}{2}\left[\left(h_{1}, k_{2}\right)-\left(h_{2}, k_{1}\right)\right]\right\},
$$

with

$$
d \mu\left(k_{1}, k_{2}\right)=\int \delta_{-2} \sum_{j=1}^{n} p_{2 J} \phi_{2 J}, 2 \sum_{j=0}^{n-1} p_{2 j+1} \phi_{2 J+1}\left(k_{1}, k_{2}\right) d \varrho_{f}(p)
$$

where $\varrho_{f}$ is the measure on $\mathbb{R}^{2 n}$, whose Fourier transform is $f$ and $p=\left(p_{1}, p_{2}, \ldots, p_{2 n}\right)$. Note that $d \mu\left(k_{1}, k_{2}\right)$ is the measure on $\mathscr{S}_{R}\left(\mathbb{R}^{s}\right) \times \mathscr{S}_{R}\left(\mathbb{R}^{s}\right)$ defined by:

$$
\int_{\mathscr{S}_{R} \times \mathscr{S}_{R}} g\left(k_{1}, k_{2}\right) d \mu\left(k_{1}, k_{2}\right)=\int_{\mathbb{R}^{2 n}} g\left(2 \sum_{j=1}^{n} p_{2 j} \phi_{2 j}, 2 \sum_{j=0}^{n-1} p_{2 j+1} \phi_{2 j-1}\right) d \varrho_{f}(p),
$$

for any bounded continuous $g$ and one has

$$
\|\mu\| \leqq \varrho_{f} \| \text {, with }\|\mu\| \text { the total variation of } \mu \text {. }
$$

We remark that the subspace $\mathfrak{M}$ contains a subset of states (cf. [11]).

We shall now show that the ultraviolet cut-off $\kappa$ in $\alpha_{t}^{\Lambda, \kappa}$ can be removed on $\mathscr{L}_{0} \cup \mathfrak{M}$. We have:

Theorem 4.1. The limit:

$$
\lim _{\kappa \rightarrow \infty} \alpha_{t}^{\Lambda, \kappa}=\alpha_{t}^{\Lambda}
$$

exists on $\mathscr{L}_{0} \cup \mathfrak{M}$ and defines an application of $\mathscr{L}_{0} \cup \mathfrak{M}$ into $B_{\alpha m}\left(\mathscr{S}_{R}\left(\mathbb{R}^{s}\right) \times \mathscr{S}_{R}\left(\mathbb{R}^{s}\right)\right.$. One has for all $h_{1}, h_{2} \in \mathscr{S}_{R}\left(R^{s}\right)$ :

$$
\begin{aligned}
\left\{\alpha_{t}^{\Lambda} E_{k_{1}, k_{2}}\right\}\left(h_{1}, h_{2}\right)= & \int_{\Omega \times \Omega} P_{\lambda|v|}^{\Lambda, t}\left(d \omega_{1}\right) P_{\lambda|v|}^{\Lambda, t}\left(d \omega_{2}\right) \\
& \cdot \exp \left\{i \Phi\left(\omega_{1}, \omega_{2}\right)\right\} \varepsilon_{k_{1}, k_{2}, t}^{\Lambda}\left(\omega_{1}, \omega_{2}\right),
\end{aligned}
$$

where

$$
\begin{aligned}
\varepsilon_{k_{1}, k_{2}}^{\Lambda} & \left(\left(m, s_{i}, x_{i}, \alpha_{i}\right),\left(n, t_{j}, y_{j^{\prime}}, \beta_{j}\right)\right) \\
= & \exp \left\{\frac{i}{2} \sum_{i \neq i^{\prime}} \alpha_{i} \alpha_{i^{\prime}} \Delta_{a}^{m}\left(s_{i}-s_{i^{\prime}}, x_{i}-x_{i^{\prime}}\right)+\frac{i}{2} \sum_{j \neq j^{\prime}} \beta_{j} \beta_{j^{\prime}} \Delta_{r}^{m}\left(t_{j}-t_{j^{\prime}}, y_{j}-y_{j^{\prime}}\right)\right\} \\
& \cdot \exp \left\{\frac{i}{2} \sum_{i, j} \alpha_{i} \beta_{j} \Delta^{m}\left(s_{i}-t_{j}, x_{i}-y_{j}\right)\right\}
\end{aligned}
$$




$$
\begin{aligned}
& \cdot \exp \left\{\frac{i}{2} \sum_{i=1}^{m} \alpha_{i}\left(\left(\partial_{0} \Delta^{m} * h_{1}\right)\left(s_{i}-t, x_{i}\right)-\left(\Delta^{m} * h_{2}\right)\left(s_{i}-t, x_{i}\right)\right)\right\} \\
& \cdot \exp \left\{-\frac{i}{2} \sum_{j=1}^{n} \beta_{j}\left(\left(\partial_{0} \Delta^{m} * h_{1}\right)\left(t_{j}-t, y_{j}\right)-\left(\Delta^{m} * h_{2}\right)\left(t_{j}-t, y_{j}\right)\right)\right\} \\
& \cdot E_{k_{1}, k_{2}}\left(h_{1,-t}+\sum_{i=1}^{m} \alpha_{i} \partial_{0} \Delta^{m}\left(s_{i}, x_{i}\right)+\sum_{j=1}^{n} \beta_{j} \partial_{0} \Delta^{m}\left(t_{j}, y_{j}\right)\right. \\
& \left.h_{2,-t}+\sum_{i=1}^{m} \alpha_{i} \Delta^{m}\left(s_{i}, x_{i}\right)+\sum_{j=1}^{n} \beta_{j} \Delta^{m}\left(t_{j}, y_{j}\right)\right)
\end{aligned}
$$

where $\Delta_{a}^{m}, \Delta_{r}^{m}$, and $\Delta^{m}$ are the invariant distributions previously defined and $\partial_{0}$ means the time derivative. $\alpha_{t}^{\Lambda}$ is a group of transformations on $\mathscr{L}_{0} \cup \mathfrak{M}$, i.e.

$$
\alpha_{s}^{\Lambda} \alpha_{t}^{\Lambda}=\alpha_{s+t}^{\Lambda} .
$$

Moreover for any $F \in \mathscr{L}_{0} \cup \mathfrak{M}$ and $h_{i} \in \mathscr{D}_{R}\left(\mathbb{R}^{s}\right), \alpha_{t}^{A}$ is a solution of the Schrödinger equation:

$$
\begin{aligned}
i \frac{\partial}{\partial t}\left\{\alpha_{t}^{\Lambda} F\right\}\left(h_{1}, h_{2}\right)= & i \int_{\mathbb{R}^{s}} d x\left\{h_{2}(x) \frac{\delta}{\delta h_{1}(x)}-h_{1}(x)\left(-\Delta+m^{2}\right) \frac{\delta}{\delta h_{2}(x)}\right\} \alpha_{t}^{\Lambda} F\left(h_{1}, h_{2}\right) \\
& -2 i \lambda \int_{\Lambda} d x \int_{\mathbb{R}} d v(\alpha) \sin \left(\frac{\alpha}{2} h_{1}(x)\right) \\
& \left.\cdot\left(\alpha_{t}^{\Lambda} F\right)\left(h_{1}, h_{2}+\alpha \delta_{x}\right)\right\},
\end{aligned}
$$

with initial condition:

$$
\lim _{t \rightarrow 0}\left(\alpha_{t}^{\Lambda} F\right)\left(h_{1}, h_{2}\right)=F\left(h_{1}, h_{2}\right) .
$$

Remark. On right hand side of (4.12) the notation $\left(\alpha_{t}^{\Lambda} F\right)\left(h_{1}, h_{2}+\alpha \delta_{x}\right)$ is of course only symbolic, since $\delta_{x} \notin \mathscr{S}\left(\mathbb{R}^{s}\right)$. The interpretation of the notation follows from the fact that $E_{k_{1}, k_{2}} \in \mathscr{L}_{0}$.

Proof. The properties on elements in $\mathscr{L}_{0} \cup \mathfrak{M}$ follow easily from those on elements of the form $E_{k_{1}, k_{2}}$. We shall now discuss the proof for these elements. From the support properties of $\Delta_{a}^{m}, \Delta_{r}^{m}$, and $\Delta^{m}$, we have $\left(\chi_{\kappa} * \Delta^{\prime} * \chi_{\kappa}\right)(0, x)=0$, where $\Delta^{\prime}$ stands for $\Delta_{a}^{m}, \Delta_{r}^{m}$, and $\Delta^{m}$.

Hence:

$$
\sum_{i, j} \alpha_{i} \alpha_{j}\left(\chi_{\kappa} * \Delta_{a}^{m} * \chi_{\kappa}\right)\left(s_{i}-s_{j}, x_{i}-x_{j}\right)=\sum_{i \neq j} \alpha_{i} \alpha_{j}\left(\chi_{\kappa} * \Delta_{a}^{m} * \chi_{\kappa}\right)\left(s_{i}-s_{j}, x_{i}-x_{j}\right),
$$

and the same for $\alpha_{i}$ replaced by $\beta_{j}$ and $\Delta_{a}^{m}$ replaced by $\Delta_{r}^{m}$. Moreover $\chi_{\kappa} * \Delta^{\prime} * \chi_{\kappa}(s, x)$ converges pointwise to $\Delta^{\prime}(s, x)$ outside of the cone $|x|=s$. Hence by the Lebesgue dominated convergence theorem we have that the expression for $\left\{\alpha_{t}^{\Lambda \kappa} E_{k_{1}, k_{2}}\right\}\left(h_{1}, h_{2}\right)$ of Theorem 3.1 converges as $\kappa \rightarrow \infty$ to the expression given by the present theorem, which we can express as a Poisson integral with respect to the measure $P_{\lambda|v|}^{\Lambda, t} \otimes P_{\lambda|v|}^{\Lambda, t}$, using Theorem 2.1. The group property is proven using the group property of $\alpha_{t}^{\Lambda \kappa}$ and the fact that $\alpha_{t}^{\Lambda} F$ is defined as the $\lim _{\kappa \rightarrow \infty} \alpha_{t}^{\Lambda, \kappa} F$ and $\alpha_{s+t}^{\Lambda} F$ 
by $\lim _{\kappa \rightarrow \infty} \alpha_{s}^{\Lambda, \kappa} \alpha_{t}^{\Lambda, \kappa} F$, which exist as seen from the fact that $\alpha_{t}^{\Lambda, \kappa}$ is defined on all $B_{\alpha m}\left(\mathscr{S}_{R}\left(\mathbb{R}^{s}\right) \times \mathscr{S}_{R}\left(\mathbb{R}^{s}\right)\right)$ and

$$
\alpha_{s}^{\Lambda} F \in B_{\alpha m}\left(\mathscr{S}_{R}\left(\mathbb{R}^{s}\right) \times \mathscr{S}_{R}\left(\mathbb{R}^{s}\right)\right)
$$

and using the explicit expression for $\alpha_{t}^{\Lambda, \kappa} G$, with $G=\alpha_{s}^{\Lambda} F$ (and once more the support properties of $\Delta^{\prime}$ and the Lebesgue dominated convergence theorem).

The Schrödinger equation is obtained using the fact that $\left(\alpha_{t}^{\Lambda, \kappa} F\right)\left(h_{1}, h_{2}\right)$ as well as $\frac{\partial}{\partial t}\left(\alpha_{t}^{\Lambda, \kappa} F\right)\left(h_{1}, h_{2}\right)$ are uniformly bounded in $\kappa$, together with the convergence of both $\left(\alpha_{t}^{\Lambda, \kappa}\right)\left(h_{1}, h_{2}\right)$ and $\frac{\partial}{\partial t} \alpha_{t}^{\Lambda, \kappa} F\left(h_{1}, h_{2}\right)$ as $\kappa \rightarrow \infty$, the compactness of the support of $h_{i}$ 's and their smoothness which allows us to interchange the time derivative and the limit $\kappa \rightarrow \infty$. The explicit form of the right hand side of (4.12) is obtained using Theorem 3.1, (3.23) and the fact that $E_{k_{1}, k_{2}} \in \mathscr{L}_{0}$.

The next step is to remove the space cut-off $\Lambda$. First we consider the case where the initial conditions are smooth and of compact support. In this case we can prove the following theorem.

Theorem 4.2. Let $\mathscr{E}$ be the restriction of the function in $\mathscr{L}_{0} \cup \mathfrak{M}$ to $\mathscr{D}_{R}\left(\mathbb{R}^{s}\right) \times \mathscr{D}_{R}\left(\mathbb{R}^{s}\right)$. Then $\lim _{\Lambda \uparrow \mathbb{R}^{s}} \alpha_{t}^{\Lambda} F=\alpha_{t} F$ exists for all $F \in \mathscr{E}$ and defines a relativistic flow on $\mathscr{E}$ in the sense that

$$
\alpha_{s} \alpha_{t}=\alpha_{s+t}, \text { for all } s, t>0 \text {. }
$$

Proof. The theorem follows immediately from the fact that the Schrödinger equation (Theorem 4.1) implies $\alpha_{t}^{\Lambda} F\left(h_{1}, h_{2}\right)=\alpha_{t}^{\Lambda^{\prime}} F\left(h_{1}, h_{2}\right)$ for all $\Lambda$ and $\Lambda^{\prime}$ containing the support of $h_{1}$.

Remark. It follows from the proof of the theorem that $\alpha_{t}$ satisfies the Schrödinger equation:

$$
\begin{aligned}
i \frac{\partial}{\partial t} \alpha_{t} F_{k_{1}, k_{2}}\left(h_{1}, h_{2}\right)= & i \int_{\mathbb{R}^{s}} d x\left\{h_{2}(x) \frac{\delta}{\delta h_{1}(x)}-h_{1}(x)\left(-\Delta+m^{2}\right) \frac{\delta}{\delta h_{2}(x)}\right\} \\
& \cdot \alpha_{t} F_{k_{1}, k_{2}}\left(h_{1}, h_{2}\right)-2 i \lambda \int_{\mathbb{R}^{s}} d x \int_{\mathbb{R}} d v(\alpha) \\
& \cdot \sin \left(\frac{\alpha}{2} h_{1}(x)\right) \alpha_{t} F_{k_{1}, k_{2}}\left(h_{1}, h_{2}+\alpha \delta_{x}\right),
\end{aligned}
$$

with initial condition $F_{k_{1}, k_{2}}\left(h_{1}, h_{2}\right)$, for any $h_{1}, h_{2} \in \mathscr{D}_{R}\left(\mathbb{R}^{s}\right)$.

The right hand side of (4.14) is equal to $P_{0}^{*} \alpha_{t} F_{k_{1}, k_{2}}\left(h_{1}, h_{2}\right)$, where $P_{0}^{*}$ is the infinitesimal generator of time translations on $\mathscr{E}$, given by the trigonometric interaction. The first term in (4.14) corresponds to the free evolution. The other generators $P_{i}^{*}$ and $M^{* i j}((i, j)=1,2,3)$ of the Poincare group associated with the same interaction are the same as in the free case [7] whereas $M^{* 0 i}$ is given by:

$$
\begin{aligned}
\left(M^{* 0 i} F\right)\left(h_{1}, h_{2}\right) & \\
= & -i \int_{\mathbb{R}^{s}} d x\left\{h_{2}(x) x^{i} \frac{\delta}{\delta h_{1}(x)}-h_{1}(x)\left(\left(-\Delta+m^{2}\right) x^{i}-\frac{\partial}{\partial x^{i}}\right) \frac{\delta}{\delta h_{2}(x)}\right\} F\left(h_{1}, h_{2}\right) \\
& -2 i \lambda \int_{\mathbb{R}^{s}} d x \int_{\mathbb{R}} d v(\alpha) x^{i} \sin \left(\frac{\alpha}{2} h_{1}(x)\right) F\left(h_{1}, h_{2}+\alpha \delta_{x}\right) .
\end{aligned}
$$


We shall now extend the existence of the infinite volume limit $\Lambda \uparrow \mathbb{R}^{s}$ to initial conditions in the larger class $\mathscr{L}_{0} \cup \mathfrak{M}$, under the assumption of small coupling constant.

Theorem 4.3. The limit

$$
\lim _{\Lambda \uparrow \mathbb{R}^{s}} \alpha_{t}^{\Lambda}=\alpha_{t}^{\infty}
$$

exists on $\mathscr{L}_{0} \cup \mathfrak{M}$ for $|\lambda|<e^{-2} c(t)^{-2}$, where $c(t)$ is a positive function of $t$ (which tends to infinity for $t \rightarrow \infty$ ).

Proof. The proof is based on the following observations

i) Formula (4.9) can be looked upon as the grand partition function of the grand canonical ensemble of a gas of two types of particles $\alpha$ and $\beta$ with the same charge distribution $d v(\alpha)$ and interacting in $s+1$ dimensions through a two-body potential $i \Delta_{r}^{m}$ (interaction of two $\alpha$-particles), $i \Delta_{a}^{m}$ (interaction of two $\beta$-particles) and $i \Delta^{m}$ (interaction of an $\alpha$-particle and a $\beta$-particle) respectively, and with a purely imaginary magnetic field.

ii) The group property for all finite $\Lambda$

$$
x_{s}^{\Lambda} x_{t}^{\Lambda}=x_{s+t}^{\Lambda}, \text { for all } s, t>0
$$

on the $E_{k_{1}, k_{2}}$ holds.

iii) For all $k_{1}, k_{2} \in \mathscr{S}_{R}\left(R^{s}\right)$

$$
\left\{\alpha_{t}^{\Lambda} E_{k_{1}, k_{2}}\right\}(0,0)=1, \text { for all } t>0 .
$$

This follows from the Schrödinger equation.

In addition the proof uses an application of the method of Kirkwood-Salzburg equations of statistical mechanics, see e.g. [36], along the lines used for ultraviolet cut-off interactions in [1]. Here we shall indicate how to define the relevant objects for the proof.

Again we remark that the convergence on arbitrary elements in $\mathscr{L}_{0} \cup \mathfrak{M}$ follows easily from the one on elements of the form $E_{k_{1}, k_{2}}$ and we discuss the proof for these elements.

Let $R_{\xi}^{v}$ be the Banach vector space of double sequences of functions:

$$
\Psi^{m, n}\left(s_{1}, x_{1}, \alpha_{1}, \ldots, s_{m}, x_{m}, \alpha_{m}, t_{1}, y_{1}, \beta_{1} \ldots t_{n}, y_{n}, \beta_{n}\right),
$$

$m, n \geqq 1, s_{i}, t_{i} \geqq 0, x_{i}, y_{i} \in \mathbb{R}^{s}, \alpha_{i}, \beta_{i} \in \mathbb{R}$, which are bounded measurable with respect to the measure:

$$
d \bar{X} d \bar{Y} d v(A) d v(B)=\prod_{i=1}^{m} d s_{i} d x_{i} d v\left(\alpha_{i}\right) \prod_{j=1}^{n} d t_{j} d y_{j} d v\left(\beta_{j}\right)
$$

and such that there exists a $\xi>0$ such that:

$$
\|\bar{\Psi}\|_{\xi}=\operatorname{Sup}_{m, n} \xi^{-(m+n)} \underset{\substack{\bar{x}_{i}, \bar{y}_{j} \\ \alpha_{i}, \beta_{j}}}{\operatorname{Sup}}\left|\Psi^{m, n}\left(\bar{x}_{i}, \alpha_{i}, \bar{y}_{j}, \beta_{j}\right)\right|,
$$

is finite, where $\bar{x}=(s, x)$ and $\bar{y}=(t, y)$. 
We define two projections in $R_{\xi}^{v}$, viz. $\Pi_{\Lambda}$ and $\Pi_{t}$ on $\Psi \in R_{\xi}^{v}$ by:

$$
\begin{array}{rlrl}
\left(\Pi_{\Lambda} \Psi\right)^{m, n}(\bar{X}, A, \bar{Y}, B) & =0, & & \text { if at least one } x_{i} \notin \Lambda, \\
& =\Psi^{m, n}(\bar{X}, A, \bar{Y}, B), & & \text { or at least one } y_{j} \notin \Lambda \\
\left(\Pi_{t} \Psi\right)^{m, n}(\bar{X}, A, \bar{Y}, B) & =0, & & \text { if at least one } s_{i} \geqq t, \\
& =\Psi^{m, n}(\bar{X}, A, \bar{Y}, B), & \text { or at least one } t_{i} \geqq t
\end{array}
$$

where for shortness $\bar{X}$ (respectively, $\bar{Y}$ ) stands for $\left\{\bar{x}_{i}\right\}_{i=1, \ldots, m}$ (respectively, $\left.\left\{\bar{y}_{i}\right\}_{i=1, \ldots, n}\right), A$ (respectively, $B$ ) for $\left\{\alpha_{i}\right\}_{i=1, \ldots, m}$ (respectively, $\left\{\beta_{j}\right\}_{j=1, \ldots, n}$ ).

Using these definitions we can rewrite the expression for $\left(\alpha_{t}^{A} E_{k_{1}, k_{2}}\right)\left(h_{1}, h_{2}\right)$ given by formulas (4.9) and (4.10) as:

$$
\begin{aligned}
\left\{\alpha_{t}^{\Lambda} E_{k_{1} k_{2}}\right\}\left(h_{1}, h_{2}\right)= & E_{k_{1}, k_{2}}\left(\alpha_{-t}\left(h_{1}, h_{2}\right)\right) \\
& \cdot\left(1+\sum_{\substack{m, n \in \mathbb{N}^{2} \\
(m, n) \neq(0,0)}} \frac{1}{m ! n !} \int d \bar{X} \int d \bar{Y} \int d v(A) \int d v(B) R_{\Lambda, t}^{m, n}(\bar{X}, A, \bar{Y}, B)\right. \\
& \left.\cdot \prod_{i \in\{1, \ldots, m\}}\left(D_{1}\left(\bar{x}_{i}, \alpha_{i}\right)-1\right) \prod_{j \in\{1, \ldots, n\}}\left(D_{2}\left(\bar{y}_{j}, \beta_{j}\right)-1\right)\right)
\end{aligned}
$$

where

$$
\begin{aligned}
R_{\Lambda, t}^{m, n}(\bar{X}, A, \bar{Y}, B)= & \sum_{r, s=0}^{\infty} \frac{\lambda^{m+n+r+s}}{r ! s !} i^{m+r-n-s} \\
& \cdot \int d \bar{X}^{\prime} \int d Y^{\prime} \int d v\left(A^{\prime}\right) \int d v\left(B^{\prime}\right) G_{\Lambda, t}\left(\bar{X} \cup \bar{X}^{\prime}, A \cup A^{\prime}, \bar{Y} \cup \bar{Y}^{\prime}, B \cup B^{\prime}\right),(
\end{aligned}
$$

and

$$
\begin{aligned}
G_{\Lambda, t}(\bar{X}, A, \bar{Y}, B)= & \exp \left(\frac{i}{2} \sum_{i=1}^{m} \sum_{j=1}^{n} \alpha_{i} \beta_{j} \Delta^{m}\left(\bar{x}_{1}-\bar{y}_{j}\right)\right) \\
& \cdot \exp \left\{\frac{i}{2} \sum_{i, j}^{m} \alpha_{i} \alpha_{j} \Delta_{a}^{m}\left(\bar{x}_{i}-\bar{x}_{j}\right)+\frac{i}{2} \sum_{k, \ell}^{n} \beta_{k} \beta_{\ell} \Delta_{r}^{m}\left(\bar{y}_{k}-\bar{y}_{\ell}\right)\right\}
\end{aligned}
$$

if all $x_{i}, y_{j} \in \Lambda$ and all $s_{i}, t_{j} \in[0, t]$ and zero otherwise. Furthermore

$$
\begin{aligned}
D_{1}(\bar{x}, \alpha)= & \exp \left\{\frac{i}{2} \alpha\left(\left(\partial_{0} \Delta^{m} * h_{1}\right)(s-t, x)-\left(\Delta^{m} * h_{2}\right)(s-t, x)\right)\right\} \\
& \cdot \exp \left\{\frac{i}{2} \alpha\left(\left(\partial_{0} \Delta^{m} * k_{2}\right)(s, x)-\left(\Delta^{m} * k_{1}\right)\left(s_{1}, x\right)\right)\right\}
\end{aligned}
$$

and

$$
\begin{aligned}
D_{2}(\bar{x}, \alpha)= & \left.\exp \left\{-\frac{i}{2} \alpha\left(\partial_{0} \Delta^{m} * h_{1}\right)(s-t, x)-\left(\Delta^{m} * h_{2}\right)(s-t, x)\right)\right\} \\
& \cdot \exp \left\{\frac{i}{2} \alpha\left(\left(\partial_{0} \Delta^{m} * k_{2}\right)(s, x)-\left(\Delta^{m} * k_{1}\right)(s, x)\right)\right\} .
\end{aligned}
$$


As an statistical mechanics we can define an operator $K$ on $R_{\xi}^{v}$ by:

$$
\begin{aligned}
\{K \Psi\}^{n^{\prime}, n}(\bar{X}, A, \bar{Y}, B)= & \exp \left\{\frac{i}{2} \sum_{j=2}^{n^{\prime}} \alpha_{1} \alpha_{j} \Delta_{a}^{m}\left(\bar{x}_{1}-\bar{x}_{j}\right)+\frac{i}{2} \sum_{k=2}^{n} \beta_{1} \beta_{k} \Delta_{r}^{m}\left(\bar{y}_{1}-\bar{y}_{j}\right)\right\} \\
& \cdot \exp \left\{\frac{i}{4} \sum_{k=1}^{n} \alpha_{1} \beta_{k} \Delta^{m}\left(\bar{x}_{1}-\bar{y}_{k}\right)+\frac{i}{4} \sum_{j=1}^{n^{\prime}} \alpha_{j} \beta_{1} \Delta^{m}\left(\bar{x}_{j}-\bar{y}_{1}\right)\right\} \\
& \cdot \Psi^{n^{\prime}-1, n-1}\left(\bar{X}, A, \bar{Y}, B \backslash\left\{\bar{x}_{1}, \alpha_{1}, \bar{y}_{1}, \beta_{1}\right\}\right)\left(1-\delta_{n^{\prime}, 1}\right)\left(1-\delta_{n, 1}\right) \\
& +\sum_{r, s=1}^{\infty} \frac{1}{r ! s !} \int d \bar{X}^{\prime} \int d \bar{Y}^{\prime} \int d v\left(A^{\prime}\right) \int d v\left(B^{\prime}\right) \\
& \cdot \prod_{i=1}^{r}\left(\exp \left\{\frac{i}{2} \alpha_{1} \alpha_{i}^{\prime} \Delta_{a}^{m}\left(\bar{x}_{1}-\bar{x}_{i}^{\prime}\right)-\frac{i}{4} \beta_{1} \alpha_{i}^{\prime} \Delta^{m}\left(\bar{y}_{1}-\bar{x}_{i}^{\prime}\right)\right\}-1\right) \\
& \cdot \prod_{j=1}^{s}\left(\exp \left\{\frac{i}{2} \beta_{1} \beta_{j}^{\prime} \Delta_{r}^{m}\left(\bar{y}_{1}-\bar{y}_{j}^{\prime}\right)+\frac{i}{4} \alpha_{1} \beta_{j}^{\prime} \Delta^{m}\left(\bar{x}_{1}-\bar{y}_{j}^{\prime}\right)\right\}-1\right) \\
& \cdot \Psi^{m+r-1, n+s-1}\left(\bar{X} \cup \bar{X}^{\prime}, A \cup A^{\prime}, \bar{Y} \cup \bar{Y}^{\prime}, B \cup B^{\prime} \backslash\left\{\bar{x}_{1}, \alpha_{1}, \bar{y}_{1}, \beta_{1}\right\}\right),
\end{aligned}
$$

where as previously $\bar{X}^{\prime}$ (respectively, $\bar{Y}^{\prime}$ ) stands for $\left\{\bar{X}_{i}^{\prime}\right\}_{i=1, \ldots, r}$ (respectively, $\left\{\bar{y}_{i}^{\prime}\right\}_{i=1, \ldots, s}$ ) and $A^{\prime}$ (respectively, $B^{\prime}$ ) for $\left\{\alpha_{i}^{\prime}\right\}_{i=1, \ldots, r}$ (respectively, $\left\{\beta_{i}^{\prime}\right\}_{i=1, \ldots, s}$ ), and $\delta_{n, k}=1$ if $n=k, \delta_{n, k}=0$ if $n \neq k$.

The norm of the operator $\Pi_{t} K \Pi_{t}$ can be readily estimated:

$$
\left\|\Pi_{t} K \Pi_{t}\right\|_{\xi} \leqq \xi^{-2} \exp \{2 \xi C(t)\},
$$

where

$$
C(t)=\operatorname{Sup}_{\alpha, \beta} \operatorname{Sup}_{\bar{y} \in[0,2 t] \times \mathbb{R} s} \int_{0}^{t} d s \int_{\mathbb{R}} d|v|(\gamma)\left|\left(\exp \left(\frac{i \alpha \gamma}{2} \Delta_{a}^{m}(\bar{x})+\frac{i \beta \gamma}{4} \Delta^{m}(\bar{x}-\bar{y})\right)-1\right)\right| .
$$

Obviously one has

$$
C(t) \leqq 4|v|(\mathbb{R}) t\left|V_{s}(t)\right|
$$

This expression is finite since for a given $\bar{y}$ it is the integral of a function bounded by $2|v|(\mathbb{R})$ over a domain which is contained in $V_{s}(t)$

$$
V_{s}(t)=\{x ;|x|<t\} \cup\{x ;|x-y|<t\} .
$$

This follows from the support properties of $\Delta^{m}, \Delta_{a}^{m}$, and $\Delta_{r}^{m}$.

The best choice of $\xi$ in view of Eq. (4.28) is

$$
\xi=C(t)^{-1},
$$

corresponding to the estimate for the norm of $\Pi_{t} K \Pi_{t}$ :

$$
\left\|\Pi_{t} K \Pi_{t}\right\| \leqq C(t)^{2} e^{2}
$$

Furthermore the operator $K$ allows us to write an integral equation for $R_{\Lambda, t}$ :

$$
R_{\Lambda, t}=\lambda^{2} \Pi_{\Lambda} \Pi_{t} S+\lambda^{2} \Pi_{\Lambda} \Pi_{t} K R_{\Lambda, t}
$$


where

$$
S_{i j}=0, \quad(i, j) \neq(1,1), \quad S_{11}=1 .
$$

Equation (4.33) can be solved by the Neumann series provided that:

$$
\lambda^{2}<C(t)^{-2} e^{-2} .
$$

With these elements the rest of the proof follows the line of [1].

We shall now give a result which shows that, at least for $\Lambda$ bounded, an asymptotics in time to a free evolution holds.

Theorem 4.4. If $m>0$, for any bounded $\Lambda \subset \mathbb{R}^{s}$, for $\lambda<\lambda_{0}$, which depends on $\Lambda$, the limit

$$
\lim _{t \rightarrow \pm \infty} \alpha_{t}^{\Lambda}=\alpha_{ \pm}^{\Lambda}
$$

exists on $\mathscr{L}_{0} \cup \mathfrak{M}$. Moreover with $F^{+, \Lambda}\left(h_{1}, h_{2}\right) \equiv \lim _{t \rightarrow+\infty}\left(\Omega_{t}^{\#} \Omega_{t}^{b}\right)\left(h_{1}, h_{2}\right)$ and $F \in \mathscr{L}_{0} \cup \mathfrak{M}, h_{1}, h_{2} \in \mathscr{S}_{R}\left(\mathbb{R}^{s}\right)$, we have: $\alpha_{ \pm}^{\Lambda} F^{+, \Lambda}=U_{t}^{0} F^{+, \Lambda}$, where $U_{t}^{0}$ is the free evolution defined in (3.15). The limit $\alpha_{ \pm}^{\Lambda} F^{+, \Lambda}$ is invariant under $\alpha_{t}^{\Lambda}$.

Proof. In a quite similar way as in the proof of Theorem 4.3 one can estimate the norm of $\Pi_{A} K \Pi_{A}$ :

$$
\left\|\Pi_{\Lambda} K \Pi_{\Lambda}\right\| \leqq c^{\prime}(\Lambda)^{2} e^{2}
$$

where

$$
c^{\prime}(\Lambda) \equiv \sup _{\alpha, \beta} \sup _{y \in \Lambda \times \mathbb{R}_{+}} \int_{\Lambda \times \mathbb{R}_{+}} d \bar{x}^{\prime} \int_{\mathbb{R}^{\prime}} d|v|\left(\alpha^{\prime}\right)\left|\exp \left(\frac{i}{2} \alpha \alpha^{\prime} \Delta_{a}^{m}\left(\bar{x}^{\prime}\right)-\frac{i}{4} \alpha^{\prime} \beta \Delta^{m}\left(\bar{x}^{\prime}-\bar{y}\right)\right)-1\right|
$$

is finite, due to the exponential decrease of $\Delta_{a}^{m}$ and $\Delta^{m}$ for large time. Consequently for $\lambda<\lambda_{0}=c^{\prime}(\Lambda)^{-1} e^{-1}$ the Kirkwood-Salzburg equation has a solution. The rest follows immediately.

Acknowledgements. We are very grateful to Prof. H. Araki for the numerous detailed valid suggestions he made to improve the presentation of this paper. We also thank the Referees who went carefully through the manuscript and pointed out a number of misprints and suggested improvements.

One of us (M.S.) wants to express his deep thanks to the members of the Physics Department of the University of Bielefeld for their warm hospitality during the Wintersemester 1980-1981 when most of this work was done. We also gratefully acknowledge support received from the Universities of AixMarseille II, Bielefeld, Bochum, Oslo and the Centre de Physique Théorique, CNRS, Marseille, during visits which made possible the completion of this work.

\section{References}

1. Albeverio, S., Høegh-Krohn, R.: Uniqueness of the physical vacuum and the Wightman functions in the infinite volume limit for some non polynomial interactions. Commun. Math. Phys. 30, 171-200 (1973)

2. Albeverio, S., Høegh-Krohn, R.: The scattering matrix for some non-polynomial interactions, I and II. Helv. Phys. Acta 46, 504-534, 536-545 (1973)

3. Albeverio, S., Høegh-Krohn, R.: Uniqueness and the global Markov property for Euclidean fields. The case of trigonometric interactions. Commun. Math. Phys. 68, 95-128 (1979)

4. Albeverio, S., Høegh-Krohn, R.: The Wightman axioms and the mass gap for strong interactions of exponential type in two-dimensional space time. J. Funct. Anal. 16, 39-82 (1974) 
5. Albeverio, S., Høegh-Krohn, R.: Mathematical theory of Feynman path integrals. In: Lecture Notes in Mathematics, Vol. 535. Berlin, Heidelberg, New York: Springer 1976

6. Albeverio, S., Høegh-Krohn, R.: Feynman path integrals and the corresponding method of stationary phase. In: Proceedings of the Conference on Feynman Path Integral, Marseille (1978). Lecture Notes in Physics, Vol. 106. Berlin, Heidelberg, New York: Springer 1979

7. Araki, H.: Hamiltonian formalism and the canonical commutation relations in quantum field theory. J. Math. Phys. 1, 492-504 (1960)

8. Araki, H.: Expansional in Banach algebras. Ann. Sci. Ec. Norm. Sup. 6, 67-84 (1973)

9. Bałaban, T., Raczka, R.: Second quantization of classical nonlinear relativistic field theory. I. Canonical formalism. J. Math. Phys. 16, 1475-1481 (1975)

Bałaban, T., Jezuita, K., Raczka, R.: Second quantization of classical nonlinear relativistic field theory. Part II. Construction of relativistic interacting local quantum field. Commun. Math. Phys. 48, 291-311 (1976)

10. Bargman, V.: On a Hilbert space of analytic functions and an associated integral transform. Part I. Commun. Pure Appl. Math. 14, 187-214 (1961)

11. Beaume, R., Manuceau, J., Pellet, A., Sirugue, M.: Translation invariant states in quantum mechanics. Commun. Math. Phys. 38, 29-45 (1974)

12. Coester, F., Haag, R. : Representation of states in a field theory with canonical variables. Phys. Rev. 117, 1137-1145 (1960)

13. Combe, Ph., Rodriguez, R., Sirugue-Collin, M.: A uniqueness theorem for anticommutation relations and commutation relations of quantum spin systems. Commun. Math. Phys. 63, 219-235 (1978)

14. Combe, Ph., Høegh-Krohn, R., Rodriguez, R., Sirugue, M., Sirugue-Collin, M. : Poisson processes on groups and Feynman path integrals. Commun. Math. Phys. 77, 269-288 (1980)

15. Combe, Ph., Høegh-Krohn, R., Rodriguez, R., Sirugue, M., Sirugue-Collin, M.: Feynman path integrals with piecewise classical paths. J. Math. Phys. 23, 405-411 (1982), and Generalized Poisson processes in quantum mechanics and field theory. Phys. Rep. 77, 221-233 (1981)

16. Combe, Ph., Høegh-Krohn, R., Rodriguez, R., Sirugue, M., Sirugue-Collin, M.: Zero-mass, 2-dimensional real sine-Gordon model without ultraviolet cut-off. Ann. Inst. H. Poincaré 37, 115-127 (1982)

17. Chebotarev, A.M., Maslov, V.P.: Jump processes and their applications in quantum mechanics, Viniti. Itogi Nauk Techn. 15, 5-78 (1978)

18. Dirac, P.A.M.: The quantum theory of the emission and absoprtion of radiation. Proc. Soc. A 114, 243-265 (1927)

19. Feldman, J.S., Osterwalder, K.: In: International symposium on mathematical problems in theoretical physics, Araki, H. (ed.). Berlin, Heidelberg, New York: Springer 1975, and The Wightman axioms and the mass gap for weakly coupled $\left(\phi^{4}\right)_{3}$ quantum field theories. Ann. Phys. 97, 80-135 (1976)

20. Friedrichs, K.O.: Mathematical aspects of the quantum theory of fields. New York: Interscience 1953

21. Friedrichs, K.O., Schapiro, L.: Integration over Hilbert space and outer extensions. Proc. Natl. Acad. Sci. 43, 336-338 (1957)

22. Fröhlich, J.: Classical and quantum statistical mechanics in one and two dimensions: twocomponent Yukawa- and Coulomb systems. Commun. Math. Phys. 47, 233-268 (1976), and In: Constructive field theory, Velo, G., Wightman, A.S. (eds.). Berlin, Heidelberg, New York: Springer 1975

23. Garding, L., Wightman, A.S. : Representations of the commutation relations. Proc. Natl. Acad. Sci. 40, 622-626 (1954)

24. Gel'fand, I.M., Yaglom, A.M.: Integration in functional spaces and its applications in quantum physics. J. Math. Phys. 1, 48-69 (1960)

25. Glimm, J., Jaffe, A.: Quantum physics. Berlin, Heidelberg, New York: Springer 1981

26. Gross, L.: In: Proceedings of conference on theory and application of analysis in function spaces, Martin, W.Ted., Segal, I.E. (eds.). Cambridge, MA: MIT Press 1972, and in Proceedings of the Vth Berkeley Symposium on Mathematical Statistics and Probability, University of California, Berkeley 1968

27. Haag, R.: On quantum field theories. Kgl. Danske Videnskab Selskab. Mat. Fys. Medd. 29, No. 12 (1955) 
28. Heisenberg, W., Pauli, W.: Zur Quantendynamik der Wellenfelder. Z. Phys. 56, 1-61 (1929); Zur Quantentheorie der Wellenfelder. II. Z. Phys. 59, 168-190 (1930)

29. van Hove, L. : Les difficultés de divergence pour un modèle particulier de champ quantifié. Physica 18, 145-159 (1957)

30. Høegh-Krohn, R.: On the spectrum of the space cut-off $P(\phi)$ : Hamiltonian in two space-time dimensions. Commun. Math. Phys. 21, 244-255 (1971)

31. Klauder, J.R.: Continuous-representation theory. I. Postulates of continuous-representation theory, and II. Generalized relation between quantum and classical dynamics. J. Math. Phys. 4, 1055-1057 and 1058-1073 (1963)

32. Magnen, J., Sénéor, R.: The infinite volume limit of the $\phi_{3}^{4}$ model. Ann. Inst. H. Poincaré 24, 95-159 (1976)

33. Moyal, J.E. : Quantum mechanics as a statistical theory. Proc. Camb. Philos. Soc. 45, 99-124 (1949)

34. von Neumann, J. : In: Collected Works, Vol. 3, Taub, A. (ed.). New York: Pergamon Press 1963

35. Polley, L., Reents, G., Streater, R.F.: Some covariant representations of massless Boson field. Preprint Darmstadt (1980)

36. Ruelle, D.: Statistical mechanics. New York: Benjamin 1969

37. Segal, I. : Distributions in Hilbert space and canonical systems of operators. Trans. Am. Math. Sọc. 88, 12-41 (1958); and Foundations of the theory of dynamical systems of infinitely many degrees of freedom. I. Kgl. Danske Videnskab Selskab. Mat. Phys. Medd. 31, No. 12 (1959)

38. Segal, I.E.: Explict formal construction of nonlinear quantum fields. J. Math. Phys. 5, 269-282 (1964)

39. Segal, I.E.: In: Proceedings of the conference on theory and applications of analysis on function space, Martin, W.Ted., Segal, I.E. (eds.). Cambridge, MA: MIT Press 1972

40. Segal, I.E. : In : Differential geometric methods in mathematics and physics, Marsden, J. (ed.). In : Lecture Notes in Mathematics. Berlin, Heidelberg, New York: Springer 1979

41. Simon, B.: The $P\left(\phi_{2}\right)$ Euclidean (quantum) field theory. Princeton, NJ : Princeton University Press 1974

42. Streater, R.F.: Canonical quantization. Commun. Math. Phys. 2, 354-374 (1966)

43. Streit, L.: A generalization of Haag's theorem. Nuovo Cimento A 10, 673-680 (1969)

44. Symanzik, K.: Euclidean quantum field theory. I. Equations for a scalar model. J. Math. Phys. 7, 510-525 (1966)

45. Umemura, Y.: Carriers of continuous measures in a Hilbertian norm. Publ. R.I.M.S. (Kyoto) A1, 1-47 and 49-54 (1965)

46. Wentzel, G.: Quantum theory of fields. New York: Interscience 1949

47. Weyl, H.: The theory of groups and quantum mechanics 2nd edn. London: Methuen 1931

48. Xia, Dao-Xing: Measure and integration on infinite dimensional spaces. New York: Academic Press 1972

Communicated by H. Araki

Received February 15, 1982; in revised form March 9, 1983 
